\title{
Hamiltonian long wave expansions for water waves over a rough bottom
}

\author{
Walter Craig * \\ Department of Mathematics and Statistics \\ McMaster University \\ Philippe Guyenne ${ }^{\dagger}$ \\ Department of Mathematics and Statistics \\ McMaster University \\ David P. Nicholls ‡ \\ Department of Mathematics \\ University of Notre Dame \\ Catherine Sulem ${ }^{\S}$ \\ Department of Mathematics \\ University of Toronto
}

\begin{abstract}
This paper is a study of the problem of nonlinear wave motion of the free surface of a body of fluid with a periodically varying bottom. The object is to describe the character of wave propagation in a long wave asymptotic regime, extending the results of R. Rosales \& G. Papanicolaou [RP]. We take the point of view of perturbation of a Hamiltonian system dependent on a small scaling parameter, with the starting point being V.E. Zakharov's Hamiltonian [Z] for the Euler equations for water waves. We consider bottom topography which is periodic in horizontal variables on a short length scale, with the amplitude of variation being of the same order as the fluid depth. The bottom may also exhibit slow variations at the same length scale as, or longer than, the order of the wavelength of the surface waves.

In the two dimensional case of waves in a channel, we give an alternate derivation of the effective $\mathrm{KdV}$ equation that is obtained in [RP]. In addition, we obtain effective Boussinesq equations that describe the motion of bidirectional long waves, in cases in which the bottom possesses both short and long scale variations. In certain cases we also obtain unidirectional equations that are similar to the $\mathrm{KdV}$ equation. In three dimensions we obtain effective three dimensional long wave equations in a Boussinesq scaling regime, and again in certain cases an effective KP system in the appropriate unidirectional limit.

The computations for these results are performed in the framework of an asymptotic analysis of multiple scale operators. In the present case this involves the DirichletNeumann operator for the fluid domain which takes into account the variations in bottom topography as well as the deformations of the free surface from equilibrium.
\end{abstract}

\footnotetext{
${ }^{*}$ Research of WC partially supported by the Canada Research Chairs Program, the NSERC through grant number 238452-01 and the NSF through grant number DMS-0070218. Part of this work was performed while a member of the MSRI, Berkeley.

${ }^{\dagger}$ Research of PG partially supported by a SHARCNET Postdoctoral Fellowship at McMaster University and the NSERC through grant number 238452-01.

${ }^{\ddagger}$ Research of DN partially supported by the NSF through grant numbers DMS-0196452 and DMS-0139822.

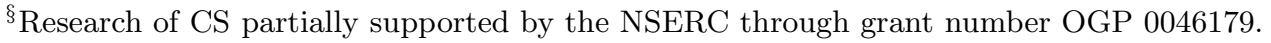




\section{Introduction}

Because of its relevance to coastal engineering, surface water wave propagation in the presence of an uneven bottom has been studied for many years. The character of coastal wave dynamics can be very complex; waves are strongly affected by the bottom through shoaling and the resulting variations in local linear wave speed, with the subsequent effects of refraction, diffraction, and reflection. Nonlinear effects, which influence waves of appreciable steepness even in the simplest of cases, have additional components due to wave/bottom as well as nonlinear wave/wave interactions, as is seen for example in depth induced breaking. The presence of bottom topography in the fluid domain introduces additional space and time scales to the classical perturbation problem. The resulting nonlinear waves can have a great influence on sediment transport and the formation of shoals and sandbars in nearshore regions, effects which are strongly felt over the longest time scales of the problem. It is therefore of central importance to understand the basic equations with which they are described.

This paper is a reassessment and extension of the work of R. Rosales \& G. Papanicolaou $[\mathrm{RP}]$ on the long wave limit of the free surface problem of water waves in the presence of a fluid domain of variable depth. It has been a general principle in the study of free surface water waves that the long-wave asymptotic scaling regime describes many of the principal aspects of the dynamics of wave motion. This is the focus of the present work, in which we study the effect of a periodic variation of the bottom on the long wavelength limit of free surface water waves. Analysing the Boussinesq scaling regime, we derive effective, or homogenised coefficients for the resulting system of nonlinear dispersive equations, which is related to the classical Boussinesq system. When considering inital data which is specifically arranged to emphasize wave motion in one direction, we derive the effective coefficients for the resulting $\mathrm{KdV}$ equation. Our analysis poses no assumptions on the amplitude nor the slope of the bottom variations; they may be considered of order $\mathcal{O}(1)$. The analysis carries through to the three dimensional case, where we derive a two-dimensional Boussinesq-like system and expressions for its effective coefficients in the first case, and a KP system in the case of wave motion in one direction alone. Because of the explicit nature of our description of the long wave perturbation analysis, and the resulting expressions for the effective coefficients of the long wave equations, we can make several general observations. First is the fact that, among bottom variations with fixed mean depth, the linear wave speed of long waves is slower than that of constant depth, strictly so for nonzero perturbations. This has previously been noted in $[\mathrm{RP}]$ in the two dimensional problem. Secondly, we give numerical computations of the effective coefficients of nonlinearity and of dispersion in typical cases. Our conclusion is that for large bottom variations, nonlinear effects dominate dispersive ones when the amplitude of bottom variations tends to the shoaling limit (with the mean depth fixed). However the balance of dispersion to nonlinearity is maintained through a remarkably large range, a fact which tends to further justify the use of the Boussinesq and $\mathrm{KdV}$ approximations in the homogenisation limit.

We extend the results of $[\mathrm{RP}]$ to cases in which the bottom varies over long spatial scales, in addition to the periodic variations on a length scale of order $\mathcal{O}(1)$. This is a class of problems which has been of interest for many years, and has generally been treated without the presence of the short-scale periodic bottom variations. The literature is very extensive, and we cite in particular the papers of R.S. Johnson [J], J.W. Miles [M], E. van Groesen \& S.R. Pudjaprasetya [GP], S.B. Yoon \& P.L.-F. Liu [YL], J.T. Kirby $[\mathrm{K}]$, the review of C.C. Mei \& P.L.-F. Liu [ML], and the books of G.B. Whitham [W] and 
A.C. Newell $[\mathrm{N}]$. In our analysis, in the case in which the bottom topography varies on the same length scale as the long waves of our scaling regime, we find a well defined Boussinesq system with effective coefficients which are functions of the slow spatial variables $X=\varepsilon x$. The three-dimensional Boussinesq regime is similar. The resulting Hamiltonian PDEs, which are nonlinear dispersive equations with variable coefficients, are quite interesting in their own right. The derivation of an effective $\mathrm{KdV}$ equation is a more subtle matter, and may be a bit artificial as significant bottom topography gives rise to scattering effects which for solutions of the water waves problem will violate any Ansatz of unidirectionality. Nonetheless there are several special situations in which one-way equations would make sense, in particular where the slow variations of the bottom topography are small, where the slow variations are of very gradual slope, or a combination of the two. We analyse these situations, giving a criterion under which a KdV scaling regime exists, and deriving the new form of the $\mathrm{KdV}$ equation when it does. In the most compelling cases, the effective equation governing unidirectional wave motion is the $\mathrm{KdV}$ equation with variable coefficients of nonlinearity and dispersion, with an additional equation for a wave component which propagates in the opposite direction, due to scattering from the variable bottom topography. The scattered wave is generated after the passage of the initial wave form; it is described by a scalar wave equation with forcing terms formed from the solution of the variable coefficient $\mathrm{KdV}$ equation. In many ways this parallels the work of $[\mathrm{GP}]$, although we augment our derivation with an estimate of the time of validity of the approximation in the various regimes of interest. There are a few differences in the conclusions as well. Our results extend to unidirectional cases in three dimensions, with the same proviso, giving rise where appropriate to KP-like systems of PDEs.

Our analysis is based on the point of view of the water waves problem as a Hamiltonian system, and we treat the perturbation problem given by the long wavelength scaling limit as a problem in systematic Hamiltonian perturbation theory. The starting point is the water waves Hamiltonian originally due to V.E. Zakharov [Z], as represented by W. Craig \& C. Sulem [CS] using the Dirichlet-Neumann operator to express the Dirichlet integral for the velocity potential over the fluid domain. The expansion of the Hamiltonian in a small parameter which governs the long wave/small amplitude asymptotic regime follows a method given in W. Craig \& M. Groves [CG] and W. Craig, P. Guyenne \& H. Kalisch [CGK]. We employ an expression for the Dirichlet-Neumann operator in the case of a variable bottom which is similar to those of Y. Liu \& D.K.P. Yue [LY] and R. Smith [S]. Using a theory of multiple scale expansions for Fourier multiplier operators given in W. Craig, C. Sulem \& P.-L. Sulem [CSS1], and results on scale separation, we identify explicit expressions for the effective coefficients of the limiting long wave problems. The fast Fourier transform is used in the numerical evaluation of these coefficients. In an appendix we give a concise derivation of the full Taylor expansion of the Dirichlet-Neumann operator in the present case, with perturbed free surface and non-constant bottom topography; it is conceivable that these expressions will be useful in other contexts, such as for numerical simulations of Euler flow.

We do not address the problem of long wave limits over random bottom topography in this paper, which is a more difficult, and probably a more important problem. Random topography is a part of the analysis of $[\mathrm{RP}]$, where effective coefficients are derived for evolution in the KdV scaling regime. The issue that arises in our approach is that the effect of scale separation is much less distinct for random bottom topography, and the statistics of bottom variations will enter in a stronger way, which will not depend simply on mean values alone. 


\section{The equations of motion}

The fluid domain consists of the region $S(\beta, \eta)=\left\{(x, y): x \in \mathbb{R}^{n-1},-h+\beta(x)<y<\eta(x)\right\}$ for spatial dimensions $n=2,3$, in which the fluid velocity is represented by the gradient of a velocity potential,

$$
u=\nabla \varphi, \quad \Delta \varphi=0
$$

where $\beta(x)$ denotes the bottom perturbation and $\eta(x)$ denotes the surface elevation. The quiescent water level is chosen at $y=0$ and the reference constant depth is represented by $h$. On the bottom boundary $\{y=-h+\beta(x)\}$, the velocity potential obeys Neumann boundary conditions

$$
\nabla \varphi \cdot N(\beta)=0
$$

where $N(\beta)=\left(1+\left|\partial_{x} \beta\right|^{2}\right)^{-1 / 2}\left(\partial_{x} \beta,-1\right)$ is the exterior unit normal. The top boundary conditions are the usual kinematic and Bernoulli conditions imposed on $\{(x, y): y=\eta(x, t)\}$, namely

$$
\partial_{t} \eta=\partial_{y} \varphi-\partial_{x} \eta \cdot \partial_{x} \varphi, \partial_{t} \varphi=-g \eta-\frac{1}{2}|\nabla \varphi|^{2} .
$$

The asymptotic analysis in this paper is from the point of view of the perturbation theory of a Hamiltonian system with respect to a small parameter. For this purpose the next section introduces the appropriate rephrasing of the above system of equations for water waves as a Hamiltonian system with infinitely many degrees of freedom.

\subsection{Hamilton's canonical equations}

The paper [Z] poses the equations of evolution (2.1), (2.2), (2.3) in the form of a Hamiltonian system in the canonical variables $(\eta(x), \xi(x))$ where one defines $\xi(x)=\varphi(x, \eta(x))$, the boundary values of the velocity potential on the free surface. The evolution equations take the classical form

$$
\partial_{t}\left(\begin{array}{l}
\eta \\
\xi
\end{array}\right)=\left(\begin{array}{cc}
0 & I \\
-I & 0
\end{array}\right)\left(\begin{array}{l}
\delta_{\eta} H \\
\delta_{\xi} H
\end{array}\right)=J \delta H
$$

with the Hamiltonian functional given by the expression

$$
\begin{aligned}
H & =\iint_{-h+\beta(x)}^{\eta(x)} \frac{1}{2}|\nabla \varphi(x, y)|^{2} d y d x+\int \frac{g}{2} \eta^{2}(x) d x \\
& =\int \frac{1}{2} \xi(x) G(\beta, \eta) \xi(x) d x+\int \frac{g}{2} \eta^{2}(x) d x .
\end{aligned}
$$

The Dirichlet-Neumann operator $G(\beta, \eta)$ is the singular integral operator which expresses the normal derivative of the velocity potential on the free surface, in terms of the boundary values $\xi(x)$ and of the domain itself, as parametrized by the functions $\beta(x)$ and $\eta(x)$ which define the lower and the upper boundaries of the fluid domain $S(\beta, \eta)$. That is, let $\varphi(x, y)$ satisfy the boundary value problem

$$
\begin{aligned}
\Delta \varphi & =0 \text { in } S(\beta, \eta), \\
\nabla \varphi \cdot N(\beta) & =0 \text { on the bottom boundary }\{y=-h+\beta(x)\}, \\
\varphi(x, \eta(x)) & =\xi(x) \text { on the free surface }\{y=\eta(x)\} .
\end{aligned}
$$

The exterior unit normal on the free surface is $N(\eta)=\left(1+\left|\partial_{x} \eta\right|^{2}\right)^{-1 / 2}\left(-\partial_{x} \eta, 1\right)$, through which the Dirichlet-Neumann operator is expressed

$$
G(\beta, \eta) \xi(x)=\nabla \varphi(x, \eta(x)) \cdot N(\eta)\left(1+\left|\partial_{x} \eta\right|^{2}\right)^{1 / 2} .
$$


It is clearly a linear operator in $\xi$, however it is nonlinear with explicitly nonlocal behavior in the two functions $\beta(x)$ and $\eta(x)$ which give the lower and upper boundaries of the fluid domain. The form of this operator, and its description in terms of the two functions $\beta(x)$ and $\eta(x)$ which define the fluid domain, are given in Section 2.2 below. The asymptotic analysis of the Dirichlet-Neumann operator $G(\beta, \eta)$ in a multiple scale regime plays a principal role in the results in this paper.

\subsection{The Dirichlet - Neumann operator}

We seek expressions for the solution of the elliptic boundary value problem (2.6) defined in the fluid domain $S(\beta, \eta)$. The principal effort of our long wave analysis of the water wave problem will be an appropriate asymptotic expansion of this operator in the presence of nontrivial bottom topography defined through $\beta(x)$, in a multiple scale regime. The bottom variations represented by $\beta(x)$ are taken to be of order $\mathcal{O}(1)$, while the surface deformations $\eta(x)$ will be small, so we will start with a description of the operator $G(\beta, 0)$, which is the case $\eta(x)=0$. When the free surface is also nonconstant, as in the situation with nontrivial solutions of the nonlinear water wave problem, a perturbation analysis for the effects of nonzero $\eta(x)$ will be used.

A central role is played by an expression for a harmonic function $\varphi(x, y)$ defined on the domain $S(\beta, 0)$, expressed in terms of its boundary data $\varphi(x, 0)=\xi(x)$ on the free surface $\{y=0\}$. In the undisturbed case, in which the bottom is flat, $\{y=-h, x \in \mathbb{R}\}$ the solution is formally given by a Fourier multiplier operator in the $x$-variables. Using the notation that $\partial_{x}=i D$;

$$
\varphi(x, y)=\iint e^{i k \cdot\left(x-x^{\prime}\right)} \frac{\cosh (k(y+h))}{\cosh (k h)} \xi\left(x^{\prime}\right) d x^{\prime} d k=\frac{\cosh ((y+h) D)}{\cosh (h D)} \xi(x) .
$$

The result is not even a tempered distribution as a function of $x \in \mathbb{R}^{n-1}$ when $y>0$, but expressions such as this are useful for our analysis, and will appear throughout this paper. The operators with which we ultimately work will however give rise to well defined distribution kernels. When the bottom topography is nontrivial, as represented by $\{y=$ $-h+\beta(x)\}$, the expression (2.8) is modified by adding a second term in order that the solution satisfies the bottom boundary conditions

$$
\varphi(x, y)=\frac{\cosh ((y+h) D)}{\cosh (h D)} \xi(x)+\sinh (y D)(L(\beta) \xi)(x)
$$

The first term in (2.9) satisfies the homogeneous Neumann condition at $y=-h$ while the second term satisfies the homogeneous Dirichlet condition at $y=0$. The operator $L(\beta)$ in the second term acts on the boundary data $\xi(x)$ given on the free surface, giving a solution to Laplace's equation in the fluid domain. When the bottom is periodic in the $x$-variables, this is tantamount to the cell problem of the method of homogenisation. Since we allow bottom perturbations to be of order $\mathcal{O}(1)$, the form of $L(\beta)$ is not explicit. An implicit description of it is given in Section 2.3.

We now turn to the expansion of the operator $G(\beta, \eta)$ for small but arbitrary perturbations $\eta(x)$ of the interface. As in the case of a flat bottom [CS, CSS2], we consider the family of 'elementary' harmonic functions in the fluid domain $S(\beta, \eta)$;

$$
\varphi_{k}(x, y)=\frac{\cosh (k(y+h))}{\cosh (k h)} e^{i k x}+\int e^{i p x} \sinh (p y) \widehat{L(\beta) e^{i k} x} d p
$$


In the calculation below, we will give the expansion of $G(\beta, \eta)$ in powers of $\eta$ (uniformly in $\beta)$. The Dirichlet-Neumann operator is defined by

$$
G(\beta, \eta) \varphi_{k}(x, \eta(x))=\partial_{y} \varphi_{k}-\left(\partial_{x} \eta\right) \partial_{x} \varphi_{k}
$$

and $G(\beta, \eta)=\sum_{l} G^{(l)}(\beta, \eta)$ where $G^{(l)}$ is of order $l$ in $\eta$. Here,

$$
\partial_{y} \varphi_{k}=k \frac{\sinh (k(h+y))}{\cosh (h k)} e^{i k x}+\int p e^{i p x} \cosh (p y) \widehat{L(\beta) e^{i k x}} d p
$$

and

$$
\partial_{x} \varphi_{k}=i k \frac{\cosh ((k(h+y))}{\cosh (h k)} e^{i k x}+\int i p e^{i p x} \sinh (p y) \widehat{L(\beta) e^{i k} x} d p
$$

At order $\mathcal{O}(1)$ in $\eta$ and $\mathcal{O}(\eta)$, one gets as predicted $G^{(0)}=D \tanh (h D)+D L(\beta)$ and $G^{(1)}=D \eta D-G^{(0)} \eta G^{(0)}$. It becomes clear that, at higher order, one gets for $G^{(l)}$ the same recursion formula as for the case of a flat bottom [CS] [CN], except that the role of the operator $G_{0}=D \tanh (h D)$ is now replaced by $G^{(0)}$.

In Section 2.3, we derive an implicit formula for the operator $L(\beta)$. In the Appendix, we give a recursion formula for $L(\beta)$ in powers of $\beta$, as well as a Taylor expansion of the Dirichlet-Neumann operator $G(\beta, \eta)$ in powers of both $\beta$ and $\eta$.

\subsection{Implicit formula for the operator $L(\beta)$}

Two dimensional case: Although the operator $L(\beta)$ is analytic for sufficiently small bottom variations $\beta(x) \in C^{1}$, we are considering variations which are of order $\mathcal{O}(1)$ and it is not a Taylor expansion in the function $\beta$ that we seek. Instead it is more useful to our methods to develop an implicit expression for $L(\beta)$ from which we can deduce information about the long wave asymptotics of the resulting operator $G(\beta, \eta)$.

Proposition 2.1. The operator $L(\beta)$ can be written in the implicit form

$$
L(\beta)=-B(\beta) A(\beta),
$$

where the operators $A(\beta)$ and $C(\beta)$ are defined by

$$
A(\beta) \xi=\int e^{i k x} \sinh (\beta(x) k) \operatorname{sech}(h k) \widehat{\xi}(k) d k, C(\beta) \xi=\int e^{i k x} \cosh ((-h+\beta(x)) k) \widehat{\xi}(k) d k
$$

and $B(\beta)=C(\beta)^{-1}$.

Proof. Using (2.9), we will employ Fourier integral expressions for the various terms appearing in the bottom boundary condition (2.2),

$$
\partial_{y} \varphi(x, y)=\int e^{i k x} k \sinh ((y+h) k) \operatorname{sech}(h k) \widehat{\xi}(k) d k+\int e^{i k x} k \cosh (y k) \widehat{L(\beta) \xi}(k) d k .
$$

The Neumann bottom boundary conditions $(2.2)$ are that $\partial_{y} \varphi-\left(\partial_{x} \beta\right) \partial_{x} \varphi=0$. The implicit formula for the operator $L(\beta)$ is derived from this condition, using the definition of $G(\beta, \eta)$ and several differential identities. In particular,

$$
\begin{aligned}
& \left.\left(D \sinh ((y+h) D) \operatorname{sech}(h D)-i\left(\partial_{x} \beta\right) D \cosh ((y+h) D) \operatorname{sech}(h D)\right) \xi\right|_{y=-h+\beta} \\
& =\int e^{i k x}\left(\sinh (\beta(x) k)-(i / k) \partial_{x}(\sinh (\beta(x) k))\right) k \operatorname{sech}(h k) \widehat{\xi}(k) d k \\
& =-i \partial_{x} \int e^{i k x} \sinh (\beta(x) k) \operatorname{sech}(h k) \widehat{\xi}(k) d k=D A(\beta) \xi
\end{aligned}
$$


The terms involving the operator $L(\beta)$ in the expression for $\partial_{y} \varphi-\left(\partial_{x} \beta\right) \partial_{x} \varphi$ are

$$
\begin{aligned}
& \left.\left(D \cosh (y D) L(\beta)-i\left(\partial_{x} \beta\right) D \sinh (y D) L(\beta)\right) \xi\right|_{y=-h+\beta} \\
& =\int e^{i k x}\left[\cosh ((-h+\beta(x)) k)-i\left(\partial_{x} \beta\right) \sinh ((-h+\beta(x)) k)\right] k \widehat{L(\beta) \xi}(k) d k \\
& =\int e^{i k x}\left[\cosh (h k)\left(\cosh (\beta(x) k)-i\left(\partial_{x} \beta\right) \sinh (\beta(x) k)\right)-\sinh (h k)(\sinh (\beta(x) k)\right. \\
& \left.\left.\quad-i\left(\partial_{x} \beta\right) \cosh (\beta(x) k)\right)\right] k \widehat{L(\beta) \xi}(k) d k \\
& =-i \partial_{x} \int e^{i k x}[\cosh (\beta(x) k) \cosh (h k)-\sinh (\beta(x) k) \sinh (h k)] \widehat{L(\beta) \xi}(k) d k \\
& =D C(\beta) L(\beta) \xi .
\end{aligned}
$$

The boundary condition involving the operator $L(\beta)$ becomes

$$
A(\beta) \xi+C(\beta) L(\beta) \xi=0
$$

which is $L(\beta)=-B(\beta) A(\beta)$.

Proposition 2.2. The inverse $B(\beta)$ of the operator $C(\beta)$ given in $(2.15)$ is well defined.

Proof. Consider the problem in the half-space $\{y<0\}$ :

$$
\Delta u=0, \quad u(x, 0)=\xi(x), \quad \partial_{y} u(x, 0)=0 .
$$

The solution of this problem is given formally by the expression $u(x, y)=\cosh (y D) \xi(x)$, and the operator $C(\beta) \xi(x)=u(x,-h+\beta(x))$ gives the trace on the curve $y=-h+\beta(x)$ of $\cosh (y D) \xi(x)$. Problem (2.19) is of course in general ill-posed. However to define $B(\beta)=$ $C(\beta)^{-1}$, one considers the alternate problem

$$
\Delta w=0 \quad \text { for } \quad(x, y) \in S(\beta, 0), \quad \partial_{y} w(x, 0)=0, \quad w(x,-h+\beta(x))=\zeta(x),
$$

which has a unique solution, and its trace on $y=0$ is well-defined. Indeed

$$
B(\beta) \zeta(x)=C(\beta)^{-1} \zeta(x)=w(x, 0) .
$$

Three dimensional case: It is straightforward to extend the formulation to three (or higher) dimensions, using $x=\left(x_{1}, x_{2}\right) \in \mathbb{R}^{2}$ to refer to the two horizontal coordinates, and retaining $y$ for the vertical coordinate. Using the notation $D=\left(D_{1}, D_{2}\right)^{T}=-i \partial_{x}=-i\left(\partial_{x_{1}}, \partial_{x_{2}}\right)^{T}$, one writes $|D|=\sqrt{\left|D_{1}\right|^{2}+\left|D_{2}\right|^{2}}$, and the first terms in the expansion of the DirichletNeumann operator are given by

$$
G^{(0)}=|D| \tanh (h|D|)+|D| L(\beta), G^{(1)}=D \cdot \eta D-G^{(0)} \eta G^{(0)} .
$$

Let us consider the configuration with an unperturbed free surface, $\eta(x)=0$. In three dimensions, the velocity potential can be expressed as

$$
\varphi(x, y)=\frac{\cosh ((y+h)|D|)}{\cosh (h|D|)} \xi(x)+\sinh (y|D|) L(\beta) \xi(x)
$$


where $\varphi(x, 0)=\xi(x)$.

Similarly as for the two-dimensional case, the operator $L(\beta)$ can be determined through the Neumann condition at the bottom The corresponding implicit formula for the operator $L(\beta)$ in three dimensions is

$$
L(\beta)=-\frac{D}{|D|} \cdot B(\beta) A(\beta) \frac{D}{|D|}
$$

where $A(\beta)=\sinh (\beta|D|) \operatorname{sech}(h|D|), \quad C(\beta)=\cosh ((\beta-h)|D|), \quad B(\beta)=C(\beta)^{-1}$.

\subsection{Integral formula for the operator $B(\beta)$}

It is of interest to notice that the operator $B(\beta)$ can be written explicitly in terms of integrals involving the Dirichlet condition $\zeta$ at the bottom and the Dirichlet-Neumann operator $G(-h+\beta)$ associated to the bottom.

Let us consider first the two-dimensional case. The fundamental solution of the Laplace equation in the half plane $\{y<0\}$ with Neumann boundary conditions is given by the method of images as

$$
\begin{aligned}
\Gamma\left(\mathrm{x}, \mathrm{x}^{\prime}\right) & =\frac{1}{2 \pi}\left(\ln \left|\mathrm{x}-\mathrm{x}^{\prime}\right|+\ln \left|\mathrm{x}-\mathrm{x}_{*}^{\prime}\right|\right) \\
& =\frac{1}{2 \pi}\left(\ln \sqrt{\left(x-x^{\prime}\right)^{2}+\left(y-y^{\prime}\right)^{2}}+\ln \sqrt{\left(x-x^{\prime}\right)^{2}+\left(y+y^{\prime}\right)^{2}}\right)
\end{aligned}
$$

where $\mathrm{x}=(x, y), \mathrm{x}^{\prime}=\left(x^{\prime}, y^{\prime}\right)$ and $\mathrm{x}_{*}^{\prime}=\left(x^{\prime},-y^{\prime}\right)$ is the reflection of $\mathrm{x}^{\prime}$ with respect to the surface plane $y=0$. Using Green's identity for a point at the surface, we have

$$
\begin{aligned}
w(x, 0)=B(\beta) \zeta(x)= & \int \nabla \Gamma\left(\mathrm{x}, \mathrm{x}^{\prime}\right) \cdot N(\beta) \sqrt{1+\left|\partial_{x^{\prime}} \beta\right|^{2}} \zeta\left(x^{\prime}\right) d x^{\prime} \\
& -\int \Gamma\left(\mathrm{x}, \mathrm{x}^{\prime}\right) G(-h+\beta) \zeta\left(x^{\prime}\right) d x^{\prime}
\end{aligned}
$$

where $N(\beta)=\left(1+\left|\partial_{x^{\prime}} \beta\right|^{2}\right)^{-1 / 2}\left(\partial_{x^{\prime}} \beta,-1\right)$ is the exterior unit normal to the bottom boundary. Substituting (2.24) into (2.25), we get:

Proposition 2.3. In two dimensions, the operator $B(\beta)$ can be written in terms of the Dirichlet condition $\zeta$ at the bottom and the Dirichlet-Neumann operator $G(-h+\beta)$ associated to the bottom in the form

$$
\begin{aligned}
B(\beta) \zeta(x)= & \frac{1}{\pi} \int \frac{\left(\partial_{x^{\prime}} \beta\right)\left(x^{\prime}-x\right)+h-\beta\left(x^{\prime}\right)}{\left(x-x^{\prime}\right)^{2}+\left(\beta\left(x^{\prime}\right)-h\right)^{2}} \zeta\left(x^{\prime}\right) d x^{\prime} \\
& -\frac{1}{2 \pi} \int \ln \left[\left(x-x^{\prime}\right)^{2}+\left(\beta\left(x^{\prime}\right)-h\right)^{2}\right] G(-h+\beta) \zeta\left(x^{\prime}\right) d x^{\prime} .
\end{aligned}
$$

In the special case $\beta=0$ (flat bottom), the formula reduces to

$$
B(0) \zeta(x)=e^{-h|D|} \zeta(x)+\tanh (h|D|) e^{-h|D|} \zeta(x)=\operatorname{sech}(h D) \zeta(x)=C(0)^{-1} \zeta(x) .
$$

A formula similar to (2.26) can be written in three dimensions. The fundamental solution of the Laplace equation is now given by

$$
\begin{aligned}
\Gamma\left(\mathrm{x}, \mathrm{x}^{\prime}\right)= & -\frac{1}{4 \pi}\left(\frac{1}{\left[\left(x_{1}-x_{1}^{\prime}\right)^{2}+\left(x_{2}-x_{2}^{\prime}\right)^{2}+\left(y-y^{\prime}\right)^{2}\right]^{1 / 2}}\right. \\
& \left.+\frac{1}{\left[\left(x_{1}-x_{1}^{\prime}\right)^{2}+\left(x_{2}-x_{2}^{\prime}\right)^{2}+\left(y+y^{\prime}\right)^{2}\right]^{1 / 2}}\right)
\end{aligned}
$$


where $\mathrm{x}=\left(x_{1}, x_{2}, y\right), \mathrm{x}^{\prime}=\left(x_{1}^{\prime}, x_{2}^{\prime}, y^{\prime}\right)$. Using the same derivation,

Proposition 2.4. In three dimensions, the operator $B(\beta)$ can be written in terms of the Dirichlet condition $\zeta$ at the bottom and the Dirichlet-Neumann operator $G(-h+\beta)$ in the form

$$
\begin{aligned}
B(\beta) \zeta\left(x_{1}, x_{2}\right)= & \frac{1}{2 \pi} \int \frac{1}{\left[\left(x_{1}-x_{1}^{\prime}\right)^{2}+\left(x_{2}-x_{2}^{\prime}\right)^{2}+(\beta-h)^{2}\right]^{1 / 2}} G(-h+\beta) \zeta d x_{1}^{\prime} d x_{2}^{\prime} \\
& -\frac{1}{4 \pi} \int \frac{\left(\partial_{x_{1}^{\prime}} \beta\right)\left(x_{1}-x_{1}^{\prime}\right)+\left(\partial_{x_{2}^{\prime}} \beta\right)\left(x_{2}-x_{2}^{\prime}\right)-h+\beta}{\left[\left(x_{1}-x_{1}^{\prime}\right)^{2}+\left(x_{2}-x_{2}^{\prime}\right)^{2}+(\beta-h)^{2}\right]^{3 / 2}} \zeta d x_{1}^{\prime} d x_{2}^{\prime} \cdot(2.29)
\end{aligned}
$$

\section{Multiple scale analysis}

We will use extensively several mathematical results on multiple scale analysis, and the behavior of Fourier multiplier operators under these scalings. Most of the analytic results have been addressed in prior work, and appear in A. Bensoussan, J.-L. Lions and G. Papanicolaou [BLP], and in particular the case of Fourier multipliers and more general pseudodifferential operators is discussed in [CSS1]. In the present context, only a subset of this analysis is required, and for the convenience of the reader we include in this section a complete presentation of what we need.

\subsection{Asymptotic expansions of multiple scale operators}

The basic Ansatz of the theory of multiple scale expansions is of a functional form $f(x, X)$ : $\mathbb{R}^{2(n-1)} \rightarrow \mathbb{C}$, where $x \in \mathbb{R}^{n-1}, X \in \mathbb{R}^{n-1}$ and $X=\varepsilon x$ is the spatial variable describing long scale variations. The dependence of a multiscale function $f$ on the short scale variable $x$ may be periodic, or possibly will be assumed to have other behavior, for example to stem from a more general stationary ergodic process. In the analysis in this paper it is important to describe the asymptotic behavior of Fourier multiplier operators on multiscale functions.

We will use the notation that $D_{x}=(1 / i) \partial_{x}$ and $D_{X}=(1 / i) \partial_{X}$. For $m=m(k)$ a function of $k \in \mathbb{R}^{n-1}$, a Fourier multiplier operator is given by

$$
m\left(D_{x}\right) f(x)=\frac{1}{(2 \pi)^{(n-1) / 2}} \int e^{i k \cdot x} m(k) \hat{f}(k) d k .
$$

Appropriate Fourier multiplier operators for our asymptotic expansions obey the standard estimates for a symbol, namely that $m(k)$ is a multiplier of order $p$ if it is smooth and satisfies

$$
\left|\partial_{k}^{\alpha} m(k)\right| \leq C_{\alpha}\left(1+|k|^{2}\right)^{\frac{p-|\alpha|}{2}} .
$$

Theorem 3.1. Let $m(k)$ be a Fourier multiplier operator of order $p$. (i) Suppose that $f(X)$ is a smooth function of the slow variables $X$. Then

$$
m\left(D_{x}\right) f(\varepsilon x)=\left(m\left(\varepsilon D_{X}\right) f\right)(\varepsilon x)=\sum_{|\alpha| \leq q} \frac{1}{\alpha !} \varepsilon^{\alpha} \partial_{k}^{\alpha} m(0)\left(D_{X}^{\alpha} f\right)(\varepsilon x)+\mathcal{O}\left(\left|\varepsilon^{q+1} D_{X}^{q+1} f\right|\right) .
$$

(ii) Suppose that $f(x, X)$ is a smooth multiscale function. Then

$$
\begin{aligned}
m\left(D_{x}\right) f(x, \varepsilon x)= & \left.\sum_{|\alpha| \leq q} \frac{1}{\alpha !} \partial_{k}^{\alpha} m\left(D_{x}\right) \varepsilon^{\alpha} D_{X}^{\alpha} f(x, X)\right|_{X=\varepsilon x} \\
& +\mathcal{O}\left(\left|\varepsilon^{q+1}\left(1+\left|D_{x}\right|^{2}\right)^{\frac{p-|q|-1}{2}} D_{X}^{q+1} f(x, X)\right|_{X=\varepsilon x} \mid\right) .
\end{aligned}
$$


Proof. In fact statement (i) follows from (ii), but it is nice to give the calculation independently for clarity of the proof. We use the expression of the Fourier transform

$$
f(\varepsilon x)=\frac{1}{(2 \pi)^{(n-1) / 2}} \int e^{i K \cdot \varepsilon x} \hat{f}(K) d K=\frac{1}{(2 \pi)^{(n-1) / 2}} \int e^{i k \cdot x} \hat{f}(k / \varepsilon) \frac{1}{\varepsilon^{n-1}} d k .
$$

Therefore the action of a Fourier multiplier on $f(\varepsilon x)$ is given by

$$
\begin{aligned}
m\left(D_{x}\right) f(\varepsilon x) & =\frac{1}{(2 \pi)^{(n-1) / 2}} \int e^{i k \cdot x} m(k) \hat{f}(k / \varepsilon) \frac{1}{\varepsilon^{n-1}} d k \\
& =\frac{1}{(2 \pi)^{(n-1) / 2}} \int e^{i \varepsilon K \cdot x} m(\varepsilon K) \hat{f}(K) d K \\
& =\frac{1}{(2 \pi)^{(n-1) / 2}} \int e^{i \varepsilon K \cdot x}\left(\sum_{|\alpha| \leq q} \frac{\varepsilon^{|\alpha|}}{\alpha !} \partial_{k}^{\alpha} m(0) K^{\alpha}+R_{q}\right) \hat{f}(K) d K
\end{aligned}
$$

This is tantamount to the expression given in (3.3). The symbol estimates on Fourier multipliers account for the estimate of the error $R_{q}$ appearing in the formula (3.3).

The calculation for statement (ii) also starts from an expression for the Fourier transform, this time for a multiscale function,

$$
f(x, \varepsilon x)=\left.f(x, X)\right|_{X=\varepsilon x}=\left.\frac{1}{(2 \pi)^{n-1}} \iint e^{i \ell \cdot x} e^{i L \cdot X}\right|_{X=\varepsilon x} \hat{f}(\ell, L) d \ell d L .
$$

Therefore the action of a Fourier multiplier is given by

$$
\begin{aligned}
\left(m\left(D_{x}\right) f\right)(x, \varepsilon x) & =\frac{1}{(2 \pi)^{n-1}} \iint e^{i k \cdot\left(x-x^{\prime}\right)} m(k) f\left(x^{\prime}\right) d x^{\prime} d k \\
& =\left.\frac{1}{(2 \pi)^{2(n-1)}} \iint e^{i k \cdot\left(x-x^{\prime}\right)} m(k) \iint e^{i \ell \cdot x^{\prime}} e^{i L \cdot X^{\prime}}\right|_{X=\varepsilon x} \hat{f}(\ell, L) d \ell d L d x^{\prime} d k \\
& =\frac{1}{(2 \pi)^{n-1}} \iint e^{i(\ell+\varepsilon L) \cdot x} m(\ell+\varepsilon L) \hat{f}(\ell, L) d \ell d L .
\end{aligned}
$$

Refering to the good behavior of the multiplier $m(k)$ under differentiation,

$$
\begin{aligned}
\left(m\left(D_{x}\right) f\right)(x, \varepsilon x) & =\frac{1}{(2 \pi)^{n-1}} \iint e^{i \ell \cdot x} e^{i L \cdot(\varepsilon x)} \sum_{\alpha \leq q} \frac{1}{\alpha !} \partial_{k}^{\alpha} m(\ell) \varepsilon^{\alpha} L^{\alpha} \hat{f}(\ell, L) d \ell d L+R_{q+1} \\
& =\left.\sum_{\alpha \leq q} \frac{1}{\alpha !} \partial_{k}^{\alpha} m\left(D_{x}\right) \varepsilon^{\alpha} D_{X}^{\alpha} f(x, X)\right|_{X=\varepsilon x}+R_{q+1}
\end{aligned}
$$

An estimate of the remainder term $R_{q+1}$ gives the result (ii) stated in (3.4).

\subsection{Scale separation}

This section develops the basic results which are used in the asymptotic expansions of multiple scale Fourier multiplier and pseudo-differential operators in this paper. The phenomenon of separation of scales is clearest in the case of periodic behavior in the short scales of the problem. 
Lemma 3.2. Suppose that the function $g(x)=g(x+\gamma)$ is continuous, and is periodic with respect to a lattice of translations $\gamma \in \Gamma \subseteq \mathbb{R}^{n-1}$, and that the function $f(X)$ is integrable and smooth. Then the short scales represented in $g(x)$ and the long scales represented by $X=\varepsilon x$ in $f(\varepsilon X)$ are asymptotically separated. That is, for all $N$ we have the estimate

$$
\int g(x) f(\varepsilon x) d x=\left.\int g(x) f(X)\right|_{X=\varepsilon x} d x=\bar{g} \int f(X) \frac{1}{\varepsilon^{n-1}} d X+\mathcal{O}\left(\varepsilon^{N}\right)
$$

where $\bar{g}=\left|\mathbb{R}^{n-1} / \Gamma\right|^{-1} \int_{\mathbb{R}^{n-1} / \Gamma} g(x) d x$.

Proof. Using the Plancherel identity,

$$
\int \overline{f(\varepsilon x)} g(x) d x=\int \overline{\hat{f}(k / \varepsilon)} \frac{1}{\varepsilon^{n-1}} \hat{g}(k) d k=\int \overline{\hat{f}(K)} \hat{g}(\varepsilon K) d K .
$$

Since $g(x)$ is periodic over a fundamental domain $\mathbb{T}^{n-1}=\mathbb{R}^{n-1} / \Gamma$, then

$$
\hat{g}(k)=\sum_{\kappa \in \Gamma^{\prime}} c_{n} \hat{g}_{\kappa} \delta(k-\kappa),
$$

where $c_{n}=\sqrt{(2 \pi)^{n-1} /\left|\mathbb{T}^{n-1}\right|}, \hat{g}_{\kappa}=\left|\mathbb{T}^{n-1}\right|^{-1 / 2} \int_{\mathbb{T}^{n-1}} e^{-i \kappa \cdot x} g(x) d x$, and we have

$$
\int \overline{\hat{f}(K)} \hat{g}(\varepsilon K) d K=\varepsilon^{-(n-1)} \sum_{\kappa \in \Gamma^{\prime}} c_{n} \overline{\hat{f}(k / \varepsilon)} \hat{g}_{\kappa}
$$

Using that $|\hat{f}(K)| \leq C_{N}\left(1+|K|^{2}\right)^{-N / 2}$ for all $N$, we have

$$
\begin{aligned}
\int \overline{\hat{f}(K)} \hat{g}(\varepsilon K) d K= & \varepsilon^{-(n-1)} \sum_{\kappa \in \Gamma^{\prime}} c_{n} \overline{\hat{f}(k / \varepsilon)} \hat{g}_{\kappa} \\
= & \varepsilon^{-(n-1)} c_{n} \hat{g}_{0} \overline{\hat{f}(0)} \\
& +\sum_{\kappa \in \Gamma^{\prime} \backslash\{0\}} \varepsilon^{-(n-1)} \overline{\hat{f}(\kappa / \varepsilon)} c_{n} \hat{g}_{\kappa} \\
= & \varepsilon^{-(n-1) \bar{g}} \int f(X) d X+\mathcal{O}\left(\varepsilon^{N}\right) .
\end{aligned}
$$

When the function $g(x, X)$ is a multiscale function itself, the analogous result is as follows.

Lemma 3.3. Suppose that $g(x, \varepsilon x)$ is continuous, and periodic in the variables $x$ of the short scales with respect to the lattice $\Gamma \subseteq \mathbb{R}^{n-1}$. For any integrable, smooth function $f(X)$ then

$$
\int_{\mathbb{R}^{n-1}} g(x, \varepsilon x) f(\varepsilon x) d x=\int \bar{g}(X) f(X) \frac{1}{\varepsilon^{n-1}} d X+\mathcal{O}\left(\varepsilon^{N}\right)
$$

where $\bar{g}(X)=\left|\mathbb{R}^{n-1} / \Gamma\right|^{-1} \int_{\mathbb{R}^{n-1} / \Gamma} g(x, X) d x$. 


\section{Long wave scaling of the Hamiltonian}

The bottom of the fluid region is allowed to vary both on a scale of order $\mathcal{O}(1)$, as well as on a slowly varying length scale. In the periodic case, this is modeled by a multiscale Ansatz on the function whose graph describes the bottom. We make no assumptions on the amplitude of $\beta$, and indeed it is allowed to be of order $\mathcal{O}(1)$. In this section, we will consider the case in which the bottom varies only on the short length scale, that is $\beta=\beta(x)$ is independent of the parameter $\varepsilon$. In the subsequent section we will extend our analysis to the more general case in which $\beta=\beta(x, X ; \varepsilon)$ where $X=\varepsilon x$, which is to say that there are variations of the bottom topography which occur on the length scale of the long waves in the problem, or possibly longer.

\subsection{The Boussinesq scaling regime}

The fundamental long wave scaling for the problem of surface water waves represents a balance between linear dispersive and nonlinear effects in the dynamics of the surface evolution. The traditional scaling that anticipates this balance is through the transformation

$$
X=\varepsilon x, \quad \varepsilon \xi^{\prime}(X)=\xi(x), \quad \varepsilon^{2} \eta^{\prime}(X)=\eta(x) .
$$

Scaling the time variable will be taken as an $\varepsilon$-dependent time change for the resulting Hamiltonian system (2.4). This represents the Boussinesq scaling regime for the problem of surface water waves in two dimensions. From the Boussinesq regime, we can recover the $\mathrm{KdV}$ equation by choice of an appropriate moving reference frame.

Introducing the scaling transformation into the Hamiltonian, we are given

$$
H\left(\eta^{\prime}, \xi^{\prime}\right)=\frac{\varepsilon^{2}}{2} \int \xi^{\prime}(\varepsilon x) G\left(\beta(x, \varepsilon x), \varepsilon^{2} \eta^{\prime}(\varepsilon x)\right) \xi^{\prime}(\varepsilon x) d x+\frac{g}{2} \int \varepsilon^{4} \eta^{\prime 2}(\varepsilon x) d x .
$$

The basic task is to compute the relevant contributions in the expansion of the DirichletNeumann operator. The two first orders in this expansion are given by

$$
\begin{aligned}
& G^{(0)}=D \tanh (h D)+D L(\beta) \\
& G^{(1)}=D \eta D-G^{(0)} \eta G^{(0)}
\end{aligned}
$$

When $L(\beta)=0$, one recovers the formulation of the water wave problem with uniform depth, whose long wave limits have been investigated in a similar manner in [CG]. In order to determine the contributions from terms involving $D L$ and since the bottom deformations are not assumed to be small, we use the implicit formula for $L(\beta)$. Note that a priori the operator $D=D_{x}+\varepsilon D_{X}$ in our two-scale problem, reducing to $D=D_{x}$ when acting on functions of $x$ alone, or to $D=\varepsilon D_{X}$ when acting on functions of $X$ alone.

Define $b(x)=\beta(x)-h$. The operators $A(\beta)$ and $C(\beta)$ which appear in the implicit formula for the operator $L(\beta)$ in (4.3), act on functions $\xi(X)$ in the long-scale variables. They can be expanded as

$$
\begin{aligned}
A(\beta)= & \sinh (\beta(x) D) \operatorname{sech}(h D)=\sinh \left(\varepsilon \beta(x) D_{X}\right) \operatorname{sech}\left(\varepsilon h D_{X}\right), \\
= & \varepsilon \beta(x) D_{X}-\frac{1}{2} \varepsilon^{3} h^{2} \beta(x) D_{X}^{3}+\frac{1}{6} \varepsilon^{3} \beta(x)^{3} D_{X}^{3}+\mathcal{O}\left(\varepsilon^{4}\right), \\
C(\beta)= & \cosh (b(x) D)=\cosh \left(b(x)\left(D_{x}+\varepsilon D_{X}\right)\right), \\
= & \cosh \left(b(x) D_{x}\right)+\varepsilon b(x) \sinh \left(b(x) D_{x}\right) D_{X} \\
+ & \frac{1}{2} \varepsilon^{2} b(x)^{2} \cosh \left(b(x) D_{x}\right) D_{X}^{2}+\frac{1}{6} \varepsilon^{3} b(x)^{3} \sinh \left(b(x) D_{x}\right) D_{X}^{3}+\mathcal{O}\left(\varepsilon^{4}\right) .
\end{aligned}
$$


From (4.6), we find for the inverse of $C(\beta)$

$$
\begin{aligned}
B(\beta)=C(\beta)^{-1}= & B_{0}(\beta)-\varepsilon B_{0}(\beta) b(x) \sinh \left(b(x) D_{x}\right) B_{0}(\beta) D_{X} \\
& -\frac{1}{2} \varepsilon^{2} B_{0}(\beta) b(x)^{2} \cosh \left(b(x) D_{x}\right) B_{0}(\beta) D_{X}^{2} \\
& +\varepsilon^{2} B_{0}(\beta) b(x) \sinh \left(b(x) D_{x}\right) B_{0}(\beta) b(x) \sinh \left(b(x) D_{x}\right) B_{0}(\beta) D_{X}^{2} \\
& -\varepsilon^{3} \frac{1}{6} B_{0}(\beta) b(x)^{3} \sinh \left(b(x) D_{x}\right) B_{0}(\beta) D_{X}^{3} \\
& +\varepsilon^{3} \frac{1}{2} B_{0}(\beta) b(x) \sinh \left(b(x) D_{x}\right) B_{0}(\beta) b(x)^{2} \cosh \left(b(x) D_{x}\right) B_{0}(\beta) D_{X}^{3} \\
& +\varepsilon^{3} \frac{1}{2} B_{0}(\beta) b(x)^{2} \cosh \left(b(x) D_{x}\right) B_{0}(\beta) b(x) \sinh \left(b(x) D_{x}\right) B_{0}(\beta) D_{X}^{3} \\
& -\varepsilon^{3} B_{0}(\beta) b(x) \sinh \left(b(x) D_{x}\right) B_{0}(\beta) b(x) \sinh \left(b(x) D_{x}\right) \\
& \times B_{0}(\beta) b(x) \sinh \left(b(x) D_{x}\right) B_{0}(\beta) D_{X}^{3}+\mathcal{O}\left(\varepsilon^{4}\right),
\end{aligned}
$$

where $B_{0}(\beta)$ stands for the inverse of the operator $\cosh \left(b(x) D_{x}\right)$, acting on functions of the short scale variables $x$. Using (4.5) and (4.7), the operator $D L(\beta)=-D B(\beta) A(\beta)$ acting on functions of the long-scale variables $\xi(X)$ can be approximated by

$$
\begin{aligned}
D L(\beta)= & -\varepsilon D_{x} B_{0}(\beta) \beta(x) D_{X} \\
& -\varepsilon^{2} B_{0}(\beta) \beta(x) D_{X}^{2}+\varepsilon^{2} D_{x} B_{0}(\beta) b(x) \sinh \left(b(x) D_{x}\right) B_{0}(\beta) \beta(x) D_{X}^{2} \\
& +\varepsilon^{3} \frac{1}{2} h^{2} D_{x} B_{0}(\beta) \beta(x) D_{X}^{3}-\varepsilon^{3} \frac{1}{6} D_{x} B_{0}(\beta) \beta(x)^{3} D_{X}^{3} \\
& +\varepsilon^{3} \frac{1}{2} D_{x} B_{0}(\beta) b(x)^{2} \cosh \left(b(x) D_{x}\right) B_{0}(\beta) \beta(x) D_{X}^{3} \\
& +\varepsilon^{3} B_{0}(\beta) b(x) \sinh \left(b(x) D_{x}\right) B_{0}(\beta) \beta(x) D_{X}^{3} \\
& -\varepsilon^{3} D_{x} B_{0}(\beta) b(x) \sinh \left(b(x) D_{x}\right) B_{0}(\beta) b(x) \sinh \left(b(x) D_{x}\right) B_{0}(\beta) \beta(x) D_{X}^{3} \\
& +\varepsilon^{4} \frac{1}{2} h^{2} B_{0}(\beta) \beta(x) D_{X}^{4}-\frac{1}{6} \varepsilon^{4} B_{0}(\beta) \beta(x)^{3} D_{X}^{4} \\
& +\varepsilon^{4} \frac{1}{2} B_{0}(\beta) b(x)^{2} \cosh \left(b(x) D_{x}\right) B_{0}(\beta) \beta(x) D_{X}^{4} \\
& -\varepsilon^{4} \frac{1}{2} h^{2} D_{x} B_{0}(\beta) b(x) \sinh \left(b(x) D_{x}\right) B_{0}(\beta) \beta(x) D_{X}^{4} \\
& +\varepsilon^{4} \frac{1}{6} D_{x} B_{0}(\beta) b(x) \sinh \left(b(x) D_{x}\right) B_{0}(\beta) \beta(x)^{3} D_{X}^{4} \\
& +\varepsilon^{4} \frac{1}{6} D_{x} B_{0}(\beta) b(x)^{3} \sinh \left(b(x) D_{x}\right) B_{0}(\beta) \beta(x) D_{X}^{4} \\
& -\varepsilon^{4} \frac{1}{2} D_{x} B_{0}(\beta) b(x) \sinh \left(b(x) D_{x}\right) B_{0}(\beta) b(x)^{2} \cosh \left(b(x) D_{x}\right) B_{0}(\beta) \beta(x) D_{X}^{4} \\
& -\varepsilon^{4} \frac{1}{2} D_{x} B_{0}(\beta) b(x)^{2} \cosh \left(b(x) D_{x}\right) B_{0}(\beta) b(x) \sinh \left(b(x) D_{x}\right) B_{0}(\beta) \beta(x) D_{X}^{4} \\
& -\varepsilon^{4} B_{0}(\beta) b(x) \sinh \left(b(x) D_{x}\right) B_{0}(\beta) b(x) \sinh \left(b(x) D_{x}\right) B_{0}(\beta) \beta(x) D_{X}^{4} \\
& +\varepsilon^{4} D_{x} B_{0}(\beta) b(x) \sinh \left(b(x) D_{x}\right) B_{0}(\beta) b(x) \sinh \left(b(x) D_{x}\right) \\
& \times B_{0}(\beta) b(x) \sinh \left(b(x) D_{x}\right) B_{0}(\beta) \beta(x) D_{X}^{4}+\mathcal{O}\left(\varepsilon^{5}\right)
\end{aligned}
$$

In a similar way, for the terms of equation (4.4) involving $D L$ in $G^{(1)}$, we obtain

$$
\begin{aligned}
D \tanh (h D) \eta D L(\beta)= & -\varepsilon^{3} D_{x}^{2} \tanh \left(h D_{x}\right) B_{0}(\beta) \beta(x) \eta^{\prime}(X) D_{X} \\
& +\varepsilon^{4} D_{x}^{2} \tanh \left(h D_{x}\right) B_{0}(\beta) b(x) \sinh \left(b(x) D_{x}\right) B_{0}(\beta) \beta(x) \eta^{\prime}(X) D_{X}^{2} \\
& -\varepsilon^{4} D_{x} \tanh \left(h D_{x}\right) B_{0}(\beta) \beta(x) \eta^{\prime}(X) D_{X}^{2} \\
& -\varepsilon^{4} h D_{x}^{2} \operatorname{sech}\left(h D_{x}\right)^{2} B_{0}(\beta) \beta(x) D_{X} \eta^{\prime}(X) D_{X} \\
& -\varepsilon^{4} D_{x} \tanh \left(h D_{x}\right) B_{0}(\beta) \beta(x) D_{X} \eta^{\prime}(X) D_{X}+\mathcal{O}\left(\varepsilon^{5}\right),
\end{aligned}
$$


and

$$
\begin{aligned}
D L \eta D L= & \varepsilon^{3} D_{x} B_{0}(\beta) \sinh \left(\beta(x) D_{x}\right) D_{x} \operatorname{sech}\left(h D_{x}\right) B_{0}(\beta) \beta(x) \eta^{\prime}(X) D_{X} \\
- & \varepsilon^{4} D_{x} B_{0}(\beta) \sinh \left(\beta(x) D_{x}\right) D_{x} \operatorname{sech}\left(h D_{x}\right) \\
& \times B_{0}(\beta) b(x) \sinh \left(b(x) D_{x}\right) B_{0}(\beta) \beta(x) \eta^{\prime}(X) D_{X}^{2} \\
+ & \varepsilon^{4} D_{x} B_{0}(\beta) \sinh \left(\beta(x) D_{x}\right) \operatorname{sech}\left(h D_{x}\right) B_{0}(\beta) \beta(x) \eta^{\prime}(X) D_{X}^{2} \\
- & \varepsilon^{4} h D_{x} B_{0}(\beta) \sinh \left(\beta(x) D_{x}\right) D_{x} \tanh \left(h D_{x}\right) \\
& \times \operatorname{sech}\left(h D_{x}\right) B_{0}(\beta) \beta(x) D_{X} \eta^{\prime}(X) D_{X} \\
+ & \varepsilon^{4} D_{x} B_{0}(\beta) \beta(x) \cosh \left(\beta(x) D_{x}\right) D_{x} \operatorname{sech}\left(h D_{x}\right) \\
& \times B_{0}(\beta) \beta(x) D_{X} \eta^{\prime}(X) D_{X} \\
- & \varepsilon^{4} D_{x} B_{0}(\beta) b(x) \sinh \left(b(x) D_{x}\right) B_{0}(\beta) \sinh \left(\beta(x) D_{x}\right) \\
& \times D_{x} \operatorname{sech}\left(h D_{x}\right) B_{0}(\beta) \beta(x) D_{X} \eta^{\prime}(X) D_{X} \\
+ & \varepsilon^{4} B_{0}(\beta) \sinh \left(\beta(x) D_{x}\right) D_{x} \operatorname{sech}\left(h D_{x}\right) \\
& \times B_{0}(\beta) \beta(x) D_{X} \eta^{\prime}(X) D_{X}+\mathcal{O}\left(\varepsilon^{5}\right) .
\end{aligned}
$$

The term $D L \eta D \tanh (h D)$ only gives first contributions at order $\mathcal{O}\left(\varepsilon^{5}\right)$ which we will not consider in this paper. Note that the contributions from $G^{(2)}$ and higher orders are also not relevant for expansions only up to order $\mathcal{O}\left(\varepsilon^{4}\right)$. Inserting the expansions for the various operators and keeping the terms up to order $\mathcal{O}\left(\varepsilon^{3}\right)$ yields the following expression for the Hamiltonian

$$
\begin{aligned}
H=\frac{1}{2} \int & -\varepsilon^{2} D_{x} B_{0}(\beta) \beta(x) \xi^{\prime}(X) D_{X} \xi^{\prime}(X)+\varepsilon^{3} g \eta^{\prime 2}(X) \\
& +\varepsilon^{3}\left(h+D_{x} B_{0}(\beta) b(x) \sinh \left(b(x) D_{x}\right) B_{0}(\beta) \beta(x)\right. \\
& \left.-B_{0}(\beta) \beta(x)\right) \xi^{\prime}(X) D_{X}^{2} \xi^{\prime}(X) d X+\mathcal{O}\left(\varepsilon^{4}\right)
\end{aligned}
$$

from which, by virtue of Lemma 3.2 on scale separation, it follows that

$$
H=\frac{\varepsilon^{3}}{2} \int\left(h+\overline{c_{1}}\right) \xi^{\prime}(X) D_{X}^{2} \xi^{\prime}(X)+g \eta^{\prime 2}(X) d X+\mathcal{O}\left(\varepsilon^{4}\right),
$$

where $c_{1}=-B_{0}(\beta) \beta(x)$. The overbar denotes the mean value over a period of the domain. In particular the term $D_{x}\left(B_{0}(\beta) \beta(x) \xi^{\prime}(X)\right)$ in (4.11) has mean value zero, being a total derivative, and it drops from the Hamiltonian at any finite order in $\varepsilon$.

Recalling from [CGK] that the scaling (4.1) modifies the symplectic structure so that $J=\varepsilon^{3} J^{\prime}$ and dropping the primes, the equations of motion can be expressed as

$$
\partial_{t} \eta=\delta_{\xi} H / \varepsilon^{3}=\left(h+\overline{c_{1}}\right) D_{X}^{2} \xi, \quad \partial_{t} \xi=-\delta_{\eta} H / \varepsilon^{3}=-g \eta,
$$

and, writing $u=\partial_{X} \xi$, they become

$$
\partial_{t} \eta=-\left(h+\overline{c_{1}}\right) \partial_{X} u, \partial_{t} u=-g \partial_{X} \eta
$$

In this linear approximation, the constant coefficient $\overline{c_{1}}$ represents the correction to uniform depth, leading to a change in wavespeed $c_{0}=\sqrt{g\left(h+\overline{c_{1}}\right)}$.

Dispersive and nonlinear effects appear when considering the next order of approximation, retaining terms up to order $\mathcal{O}\left(\varepsilon^{5}\right)$. It is clear that all terms of order $\mathcal{O}\left(\varepsilon^{3}\right)$, as well as all terms which have the first factor which is a derivative $D_{x}$ in (4.8), (4.9) and (4.10), will 
not contribute to the Hamiltonian by virtue of the lemma on scale separation, as they have

zero mean value on the periodic fundamental domain $\mathbb{T}^{n-1}$. The Hamiltonian then reads

$$
\begin{aligned}
H=\frac{\varepsilon^{3}}{2} \int & \left(h+\overline{c_{1}}\right) \xi^{\prime}(X) D_{X}^{2} \xi^{\prime}(X)+g \eta^{2}(X) \\
& +\varepsilon^{2}\left(1+\overline{c_{2}}\right) \xi^{\prime}(X) D_{X} \eta^{\prime}(X) D_{X} \xi^{\prime}(X) \\
& -\varepsilon^{2}\left(\overline{c_{3}}+\frac{1}{3} h^{3}\right) \xi^{\prime}(X) D_{X}^{4} \xi^{\prime}(X) d X+\mathcal{O}\left(\varepsilon^{7}\right),
\end{aligned}
$$

with

$$
c_{2}=-B_{0}(\beta) \sinh \left(\beta(x) D_{x}\right) D_{x} \operatorname{sech}\left(h D_{x}\right) B_{0}(\beta) \beta(x),
$$

and

$$
\begin{aligned}
c_{3}= & -\frac{1}{2} h^{2} B_{0}(\beta) \beta(x)+\frac{1}{6} B_{0}(\beta) \beta(x)^{3}-\frac{1}{2} B_{0}(\beta) b(x)^{2} \beta(x) \\
& +B_{0}(\beta) b(x) \sinh \left(b(x) D_{x}\right) B_{0}(\beta) b(x) \sinh \left(b(x) D_{x}\right) B_{0}(\beta) \beta(x) .
\end{aligned}
$$

In terms of $u^{\prime}=\partial_{X} \xi^{\prime}$ (and dropping the primes), the corresponding equations of motion are given by

$$
\begin{aligned}
\partial_{t} \eta & =-\partial_{X}\left(\left(h+\overline{c_{1}}+\varepsilon^{2}\left(1+\overline{c_{2}}\right) \eta\right) u\right)-\varepsilon^{2}\left(\overline{c_{3}}+\frac{1}{3} h^{3}\right) \partial_{X}^{3} u \\
\partial_{t} u & =-g \partial_{X} \eta-\varepsilon^{2}\left(1+\overline{c_{2}}\right) u \partial_{X} u
\end{aligned}
$$

which constitute extensions of the Boussinesq equations to the case of a varying depth. The time of validity of the approximation given by these equations is at least of order $\mathcal{O}\left(\varepsilon^{-2}\right)$, which is the same as the problem with a bottom to the fluid domain of uniform $\operatorname{depth} \beta(x)=0$. When $\beta(x)=0$, or otherwise when the coefficients $\overline{c_{1}}, \overline{c_{2}}$ and $\overline{c_{3}}$ vanish, equations (4.18) reduce to the Boussinesq equations for a uniform depth.

\subsection{The $\mathrm{KdV}$ limit}

In this section, we adopt the procedure given in [CG] to derive a unidirectional analog to equations (4.18). Starting from the Hamiltonian (4.15) in the form

$$
\begin{gathered}
H=\frac{1}{2} \int \varepsilon^{3} g \eta^{\prime 2}(X)+\varepsilon^{3}\left(h+\overline{c_{1}}\right) u^{\prime 2}(X)+\varepsilon^{5}\left(1+\overline{c_{2}}\right) \eta^{\prime}(X) u^{\prime 2}(X) \\
-\varepsilon^{5}\left(\overline{c_{3}}+\frac{1}{3} h^{3}\right)\left(\partial_{X} u^{\prime}(X)\right)^{2} d X+\mathcal{O}\left(\varepsilon^{7}\right)
\end{gathered}
$$

we introduce the new variables $r$ and $s$ such that

$$
\eta^{\prime}=\sqrt[4]{\frac{\left(h+\overline{c_{1}}\right)}{4 g}}(r+s), \quad u^{\prime}=\sqrt[4]{\frac{g}{4\left(h+\overline{c_{1}}\right)}}(r-s) .
$$

The Hamiltonian becomes

$$
\begin{aligned}
H=\frac{1}{2} & \int \varepsilon^{3} \sqrt{g\left(h+\overline{c_{1}}\right)}\left(r^{2}+s^{2}\right)+\varepsilon^{5}\left(\frac{1+\overline{c_{2}}}{2}\right) \sqrt[4]{\frac{g}{4\left(h+\overline{c_{1}}\right)}}\left(r^{3}-r^{2} s-r s^{2}+s^{3}\right) \\
& -\varepsilon^{5}\left(\overline{c_{3}}+\frac{1}{3} h^{3}\right) \sqrt{\frac{g}{4\left(h+\overline{c_{1}}\right)}}\left(\left(\partial_{X} r\right)^{2}-2\left(\partial_{X} r\right)\left(\partial_{X} s\right)+\left(\partial_{X} s\right)^{2}\right) d X
\end{aligned}
$$


It is also useful to transform the system into a coordinate frame moving with the characteristic velocity $c_{0}=\sqrt{g\left(h+\overline{c_{1}}\right)}$, which is effected by subtracting the conserved momentum integral

$$
c_{0} I=\varepsilon^{3} c_{0} \int \eta^{\prime} u^{\prime} d X=\frac{1}{2} \varepsilon^{3} \sqrt{g\left(h+\overline{c_{1}}\right)} \int\left(r^{2}-s^{2}\right) d X
$$

from the Hamiltonian. This yields

$$
\begin{aligned}
H-c_{0} I=\frac{1}{2} & \int 2 \varepsilon^{3} \sqrt{g\left(h+\overline{c_{1}}\right)} s^{2}+\varepsilon^{5}\left(\frac{1+\overline{c_{2}}}{2}\right) \sqrt[4]{\frac{g}{4\left(h+\overline{c_{1}}\right)}}\left(r^{3}-r^{2} s-r s^{2}+s^{3}\right) \\
& -\varepsilon^{5}\left(\overline{c_{3}}+\frac{1}{3} h^{3}\right) \sqrt{\frac{g}{4\left(h+\overline{c_{1}}\right)}}\left(\left(\partial_{X} r\right)^{2}-2\left(\partial_{X} r\right)\left(\partial_{X} s\right)+\left(\partial_{X} s\right)^{2}\right) d X(4.23)
\end{aligned}
$$

The evolution equations for $r$ and $s$ can now be written as

$$
\partial_{t} r=-\partial_{X} \delta_{r}\left(H-c_{0} I\right) / \varepsilon^{3}, \quad \partial_{t} s=\partial_{X} \delta_{s}\left(H-c_{0} I\right) / \varepsilon^{3} .
$$

In explicit terms, this is the following system of two coupled equations

$$
\begin{aligned}
\partial_{t} r= & -\varepsilon^{2}\left(\frac{1+\overline{c_{2}}}{4}\right) \sqrt[4]{\frac{g}{4\left(h+\overline{c_{1}}\right)}} \partial_{X}\left(3 r^{2}-2 r s-s^{2}\right) \\
& -\varepsilon^{2}\left(\overline{c_{3}}+\frac{1}{3} h^{3}\right) \sqrt{\frac{g}{4\left(h+\overline{c_{1}}\right)}}\left(\partial_{X}^{3} r-\partial_{X}^{3} s\right), \\
\partial_{t} s= & 2 \sqrt{g\left(h+\overline{c_{1}}\right)} \partial_{X} s-\varepsilon^{2}\left(\frac{1+\overline{c_{2}}}{4}\right) \sqrt[4]{\frac{g}{4\left(h+\overline{c_{1}}\right)}} \partial_{X}\left(r^{2}+2 r s-3 s^{2}\right) \\
& -\varepsilon^{2}\left(\overline{c_{3}}+\frac{1}{3} h^{3}\right) \sqrt{\frac{g}{4\left(h+\overline{c_{1}}\right)}}\left(\partial_{X}^{3} r-\partial_{X}^{3} s\right) .
\end{aligned}
$$

The solution $r$ corresponds to a predominantly right-moving component, while $s$ corresponds to a predominantly left-moving component.

The KdV regime consists in restricting one's attention to regions of the phase space where the equation for $r$ decouples to lowest order from the equation for $s$. Concentrating on the region of the phase space that corresponds to a predominantly right-moving evolution (this will be the region where $s$ remains of order $\mathcal{O}\left(\varepsilon^{2}\right)$ ), all terms but those which depend on $r$ alone in the right-hand side of (4.25) are of higher order in $\varepsilon$ and thus can be neglected. It has been proved in Craig [C] that this regime is valid over time intervals of order $\mathcal{O}\left(\varepsilon^{-2}\right)$ if the initial conditions are appropriately chosen. Setting $T=\varepsilon^{2} t$, which is a time change for the Hamiltonian, we have a closed equation for the variable $r$, namely

$$
\partial_{T} r=-3\left(\frac{1+\overline{c_{2}}}{2}\right) \sqrt[4]{\frac{g}{4\left(h+\overline{c_{1}}\right)}} r \partial_{X} r-\left(\overline{c_{3}}+\frac{1}{3} h^{3}\right) \sqrt{\frac{g}{4\left(h+\overline{c_{1}}\right)}} \partial_{X}^{3} r
$$

which is the $\mathrm{KdV}$ equation whose coefficients are modified to account for the rapidly varying depth. When $\overline{c_{1}}, \overline{c_{2}}$ and $\overline{c_{3}}$ are zero, equation (4.27) reduces to the classical KdV equation.

\subsection{Properties of the coefficients}

The coefficients $\overline{c_{1}}, \overline{c_{2}}$ and $\overline{c_{3}}$ derived in Section 4.1 are of central concern to understand the effects of bottom topography on long wave evolution of the free surface problem. They are given by explicit expressions which are functionals of the perturbation $\beta(x)$ of the 
bottom. To fix a reference depth, we normalize $\int_{0}^{2 \pi} \beta(x) d x=0$. In this section, we give an alternate proof of the fact that $\overline{c_{1}} \leq 0$, which was remarked in $[\mathrm{RP}]$. That is, the presence of nontrivial topography results in a smaller velocity $\sqrt{g\left(h+\overline{c_{1}}\right)}$ for linear evolution of long waves. In addition, we compute numerically the coefficients $\overline{c_{1}}, \overline{c_{2}}$ and $\overline{c_{3}}$ for the particular perturbation $\beta(x)=\beta_{0} \cos (x)$ as functions of the amplitude $\beta_{0}\left(0 \leq \beta_{0} \leq h\right)$. We also evaluate the ratio

$$
R=\frac{3}{2}\left(\frac{1+\overline{c_{2}}}{\overline{c_{3}}+\frac{1}{3} h^{3}}\right) \sqrt[4]{\frac{4\left(h+\overline{c_{1}}\right)}{g}},
$$

of the coefficients of nonlinearity to dispersion in the $\mathrm{KdV}$ equation which determines the dominant effect as $\beta_{0}$ increases.

Proposition 4.1. For bottom perturbations $\beta(x)$ with zero mean value, the effective depth $\bar{h}=h+\overline{c_{1}}$ satisfies $\bar{h} \leq h$, with equality only if $\beta(x)=0$.

Proof. Let $\beta(x)$ be of zero mean value and consider $w$ a solution of the problem

$$
\Delta w=0 \text { in } S(\beta, 0), \quad \partial_{y} w=0 \text { on } y=0, \quad w(x,-h+\beta(x))=\beta(x) .
$$

By the definition of $B_{0}(\beta) \beta$, we have $\overline{c_{1}}=-\int_{0}^{2 \pi} w(x, 0) d x$. Firstly,

$$
0=\iint_{S(\beta, 0)} \Delta w d x d y=\int_{0}^{2 \pi} \partial_{y} w(x, 0) d x-\int_{y=-h+\beta} \partial_{N} w d \sigma
$$

so we conclude that $\int_{y=-h+\beta} \partial_{N} w d \sigma=0$. Secondly, integrating the function $(w(x, y)-$ $y) \Delta w$ over $S(\beta, 0)$, we find

$$
\begin{aligned}
& \iint_{S(\beta, 0)}|\nabla w|^{2} d x d y-\iint_{S(\beta, 0)} \partial_{y} w d x d y \\
= & \int_{0}^{2 \pi}(w-y) \partial_{y} w(x, 0) d x+\int_{y=-h+\beta}(w-y) \partial_{N} w d \sigma .
\end{aligned}
$$

Since $\partial_{y} w(x, 0)=0$ and $\left.(w-y)\right|_{y=-h+\beta}=h$, both terms in the RHS vanish and we have

$$
\iint_{S(\beta, 0)}|\nabla w|^{2} d x d y=\iint_{S(\beta, 0)} \partial_{y} w d x d y=\int_{0}^{2 \pi} w(x, 0) d x-\int_{0}^{2 \pi} \beta(x) d x .
$$

Because $\beta(x)$ has zero mean value, the quantity $\int_{0}^{2 \pi} w(x, 0) d x \geq 0$, and vanishes only when $w(x, y)$ is identically zero. We remark that the same conclusion $\overline{c_{1}}<0$ holds in arbitrary spatial dimensions.

The numerical calculations of $\overline{c_{1}}, \overline{c_{2}}$ and $\overline{c_{3}}$ are performed using a Fourier spectral method. The Fourier multipliers involving $\beta(x) D_{x}$ are consistently expanded up to third order in $\beta$, and explicit Fourier multiplier operations are performed using Fast Fourier Transforms. Typically, we chose a number of modes equal to 512 and $g=h=1$. Figure 1 shows graphs of the coefficients $h+\overline{c_{1}}, 1+\overline{c_{2}}$ and $\frac{1}{3} h^{3}+\overline{c_{3}}$. We note that all three coefficients decrease with increasing $\beta_{0}$. The interpretation is that the time scale describing the $\mathrm{KdV}$ regime is slower with increasing $\beta_{0}$. Better approximations to $\overline{c_{1}}, \overline{c_{2}}$ and $\overline{c_{3}}$ can be obtained for $\beta_{0}$ close to $h$ by including higher-order terms, but this would not change qualitatively the results. 

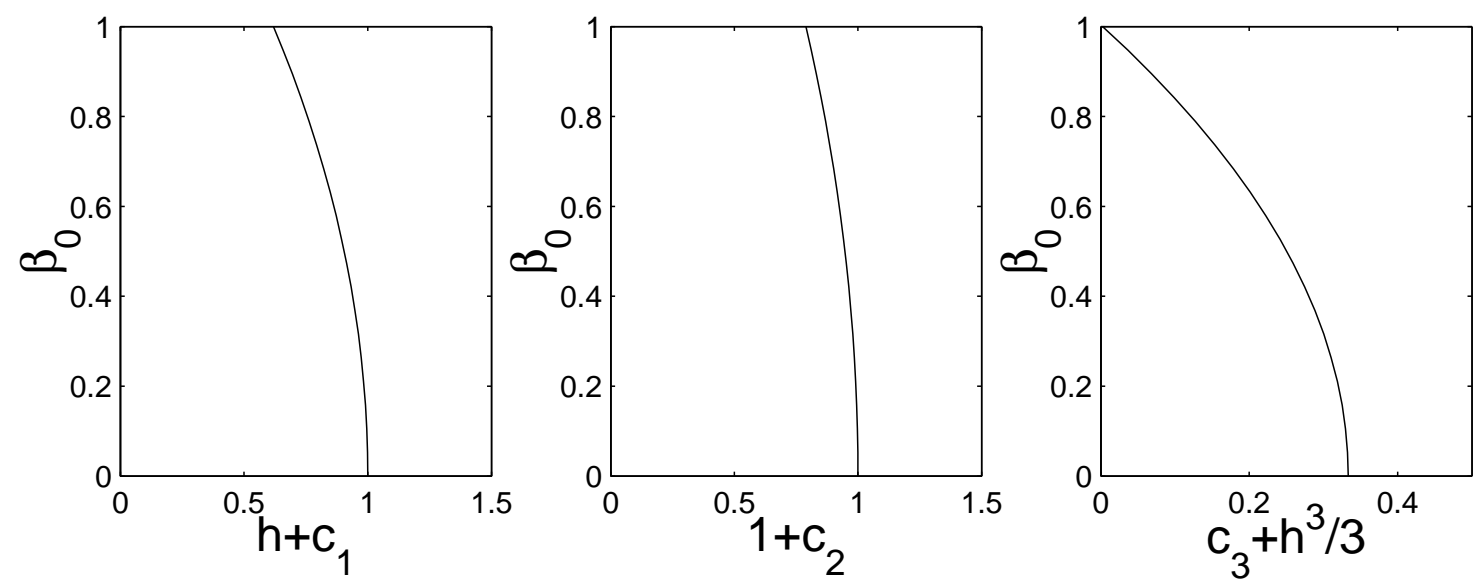

Figure 1: Coefficients $h+\overline{c_{1}}, 1+\overline{c_{2}}$ and $\overline{c_{3}}+\frac{1}{3} h^{3}$ as functions of $\beta_{0}$. The bottom perturbation is $\beta(x)=\beta_{0} \cos (x)$. Computations are performed with a number of modes equal to 512 and $h=1$. Expressions of $\overline{c_{1}}, \overline{c_{2}}$ and $\overline{c_{3}}$ are expanded up to third order in $\beta$.

Figure 2 shows the ratio $R$ between the effective coefficients of nonlinearity and dispersion in the resulting $\mathrm{KdV}$ equation (4.27). We observe that nonlinear effects dominate dispersive effects as $\beta_{0}$ approaches $h$. However, the two effects seem to be remarkably of the same order of magnitude until $\beta_{0} \simeq 0.7 h$. As $\beta_{0} / h$ approaches unity, the ratio diverges. Therefore, the KdV regime, in which dispersive and nonlinear effects are balanced, remains valid for a significant range of $\beta_{0}$.

\subsection{Long-wave approximation to the three-dimensional problem}

Two kinds of long-wave scalings are usually possible: ones which are isotropic in both horizontal directions and ones which have different length scales in the $x_{1}$ - and $x_{2}$-directions. We will focus on the latter in this section. A common nonisotropic spatial scaling regime deals with wave motions which are long in one horizontal direction and very long in the other. For this purpose, we introduce spatial and amplitude scalings as follows

$$
X_{1}=\varepsilon x_{1}, \quad X_{2}=\varepsilon^{2} x_{2}, \quad \varepsilon \xi^{\prime}\left(X_{1}, X_{2}\right)=\xi\left(x_{1}, x_{2}\right), \quad \varepsilon^{2} \eta^{\prime}\left(X_{1}, X_{2}\right)=\eta\left(x_{1}, x_{2}\right) .
$$

Let us consider first the case where the bottom only varies on a single length scale $\mathcal{O}(1)$ in both horizontal directions, namely $\beta=\beta\left(x_{1}, x_{2}\right)$. Again, we do not assume any smallness of the amplitude of $\beta$. The same procedure as in two dimensions is applied to expand the Hamiltonian in powers of the small parameter $\varepsilon$. We will use the implicit formula for $L(\beta)$ in three dimensions. In the first approximation, the Hamiltonian is written as

$$
H=\frac{\varepsilon^{3}}{2} \int\left(h+\overline{c_{1}}\right) \xi^{\prime} D_{X_{1}}^{2} \xi^{\prime}+g \eta^{2} d X_{1} d X_{2}+\mathcal{O}\left(\varepsilon^{5}\right)
$$

where $c_{1}=-B_{0}(\beta) \beta$. As above, $B_{0}(\beta)$ stands for the inverse of the operator $\cosh (b|D|)$ and $b\left(x_{1}, x_{2}\right)=\beta\left(x_{1}, x_{2}\right)-h$. Dropping the primes, the corresponding equations of motion are given by

$$
\partial_{t} \eta=\delta_{\xi} H / \varepsilon^{3}=-\left(h+\overline{c_{1}}\right) \partial_{X_{1}}^{2} \xi, \quad \partial_{t} \xi=-\delta_{\eta} H / \varepsilon^{3}=-g \eta,
$$




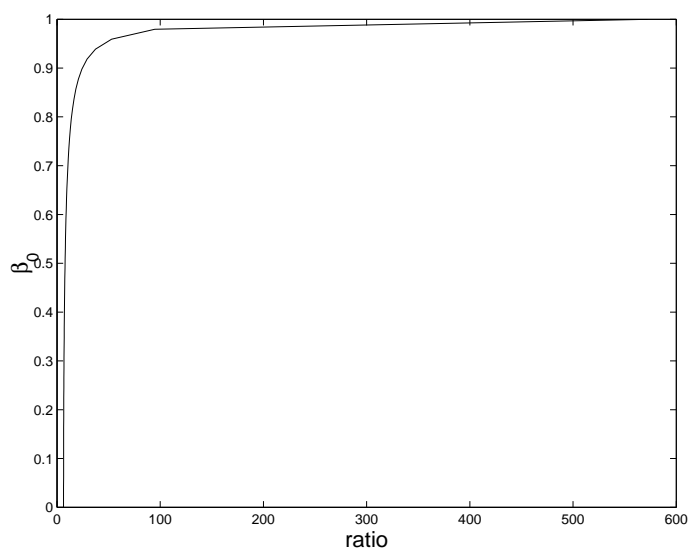

Figure 2: Ratio $R$ of nonlinear to dispersive coefficients as a function of $\beta_{0}$. Computations are performed with a number of modes equal to 512 and $g=h=1$.

which coincide with the linear approximation that we derived for the two-dimensional equations. At the second order approximation, we have

$$
\begin{aligned}
H= & \frac{\varepsilon^{3}}{2} \int\left(h+\overline{c_{1}}\right) \xi^{\prime} D_{X_{1}}^{2} \xi^{\prime}+g \eta^{\prime 2}+\varepsilon^{2}\left(1+\overline{c_{2}}\right) \xi^{\prime} D_{X_{1}} \eta^{\prime} D_{X_{1}} \xi^{\prime} \\
& -\varepsilon^{2}\left(\overline{c_{3}}+\frac{1}{3} h^{3}\right) \xi^{\prime} D_{X_{1}}^{4} \xi^{\prime}+\varepsilon^{2}\left(h+\overline{c_{1}}\right) \xi^{\prime} D_{X_{2}}^{2} \xi^{\prime} d X_{1} d X_{2}+\mathcal{O}\left(\varepsilon^{7}\right),
\end{aligned}
$$

with

$$
\begin{aligned}
c_{2}= & -B_{0}(\beta) \sinh (\beta|D|) D_{x_{1}}^{2}|D|^{-1} \operatorname{sech}(h|D|) B_{0}(\beta) \beta \\
& -B_{0}(\beta) \sinh (\beta|D|) D_{x_{1}} D_{x_{2}}|D|^{-1} \operatorname{sech}(h|D|) B_{0}(\beta) \beta,
\end{aligned}
$$

and

$$
\begin{aligned}
c_{3}= & -\frac{1}{2} h^{2} B_{0}(\beta) \beta+\frac{1}{6} B_{0}(\beta) \beta^{3}-\frac{1}{2} B_{0}(\beta) b^{2} \cosh (b|D|) D_{x_{1}}^{2}|D|^{-2} B_{0}(\beta) \beta \\
& +B_{0}(\beta) b \sinh (b|D|) D_{x_{1}}|D|^{-1} B_{0}(\beta) b \sinh (b|D|) D_{x_{1}}|D|^{-1} B_{0}(\beta) \beta \\
& -\frac{1}{2} B_{0}(\beta) b \sinh (b|D|)\left(1-D_{x_{1}}^{2}|D|^{-2}\right)|D|^{-1} B_{0}(\beta) \beta .
\end{aligned}
$$

Note here that $|D|=\sqrt{\left|D_{x_{1}}\right|^{2}+\left|D_{x_{2}}\right|^{2}}$. The evolution of $\eta$ and $\xi$ is now governed by

$$
\begin{aligned}
\partial_{t} \eta & =-\partial_{X_{1}}\left(\left(h+\overline{c_{1}}+\varepsilon^{2}\left(1+\overline{c_{2}}\right) \eta\right) \partial_{X_{1}} \xi\right)-\varepsilon^{2}\left(\overline{c_{3}}+\frac{1}{3} h^{3}\right) \partial_{X_{1}}^{4} \xi-\varepsilon^{2}\left(h+\overline{c_{1}}\right) \partial_{X_{2}}^{2} \xi \\
\partial_{t} \xi & =-g \eta-\frac{1}{2} \varepsilon^{2}\left(1+\overline{c_{2}}\right)\left(\partial_{X_{1}} \xi\right)^{2} .
\end{aligned}
$$

\subsection{The KP equation}

The KP equation is a three-dimensional analog of the KdV equation, which is derived under the assumption that transverse variations of the wave motions in the $x_{2}$-direction are weaker than those in the $x_{1}$-direction. Its derivation in the present Hamiltonian framework is carried out by means of the same technique as that described in Section 4.2. Let us express the Hamiltonian (4.33) in terms of $u^{\prime}=\partial_{X_{1}} \xi^{\prime}$,

$$
\begin{aligned}
H= & \frac{\varepsilon^{3}}{2} \int\left(h+\overline{c_{1}}\right) u^{\prime 2}+g \eta^{\prime 2}+\varepsilon^{2}\left(1+\overline{c_{2}}\right) \eta^{\prime} u^{\prime 2} \\
& \quad-\varepsilon^{2}\left(\overline{c_{3}}+\frac{1}{3} h^{3}\right)\left(\partial_{X_{1}} u^{\prime}\right)^{2}+\varepsilon^{2}\left(h+\overline{c_{1}}\right)\left(\partial_{X_{1}}^{-1} \partial_{X_{2}} u^{\prime}\right)^{2} d X_{1} d X_{2}+\mathcal{O}\left(\varepsilon^{7}\right) .
\end{aligned}
$$


Making the further change of variables

$$
\eta^{\prime}=\sqrt[4]{\frac{\left(h+\overline{c_{1}}\right)}{4 g}}(r+s), \quad u^{\prime}=\sqrt[4]{\frac{g}{4\left(h+\overline{c_{1}}\right)}}(r-s),
$$

one arrives at

$$
\begin{aligned}
H=\frac{\varepsilon^{3}}{2} & \int \sqrt{g\left(h+\overline{c_{1}}\right)}\left(r^{2}+s^{2}\right)+\varepsilon^{2}\left(\frac{1+\overline{c_{2}}}{2}\right) \sqrt[4]{\frac{g}{4\left(h+\overline{c_{1}}\right)}}\left(r^{3}-r^{2} s-r s^{2}+s^{3}\right) \\
& -\varepsilon^{2}\left(\overline{c_{3}}+\frac{1}{3} h^{3}\right) \sqrt{\frac{g}{4\left(h+\overline{c_{1}}\right)}}\left(\left(\partial_{X_{1}}(r-s)\right)^{2}\right. \\
& +\varepsilon^{2}\left(\frac{h+\overline{c_{1}}}{2}\right) \sqrt{\frac{g}{h+\overline{c_{1}}}}\left(\partial_{X_{1}}^{-1} \partial_{X_{2}}(r-s)\right)^{2} d X_{1} d X_{2}
\end{aligned}
$$

Subtracting the momentum integral (4.22) and restricting the phase space to an $\varepsilon^{2}$-neighborhood of $\eta \sim u$ as in the two-dimensional case, the Hamiltonian reduces to

$$
\begin{gathered}
H-c_{0} I=\frac{\varepsilon^{3}}{2} \int \varepsilon^{2}\left(\frac{1+\overline{c_{2}}}{2}\right) \sqrt[4]{\frac{g}{4\left(h+\overline{c_{1}}\right)}} r^{3}-\varepsilon^{2}\left(\overline{c_{3}}+\frac{1}{3} h^{3}\right) \sqrt{\frac{g}{4\left(h+\overline{c_{1}}\right)}}\left(\partial_{X_{1}} r\right)^{2} \\
+\varepsilon^{2}\left(\frac{h+\overline{c_{1}}}{2}\right) \sqrt{\frac{g}{h+\overline{c_{1}}}}\left(\partial_{X_{1}}^{-1} \partial_{X_{2}} r\right)^{2} d X_{1} d X_{2}
\end{gathered}
$$

which is valid up to order $\mathcal{O}\left(\varepsilon^{5}\right)$. One can write a system of equations for $r$ and $s$, in terms of a slow time variable $T=\varepsilon^{2} t$, as

$$
\begin{aligned}
\partial_{T} r= & -\partial_{X_{1}} \delta_{r}\left(H-c_{0} I\right) / \varepsilon^{5}=-3\left(\frac{1+\overline{c_{2}}}{2}\right) \sqrt[4]{\frac{g}{4\left(h+\overline{c_{1}}\right)}} r \partial_{X_{1}} r \\
& -\left(\overline{c_{3}}+\frac{1}{3} h^{3}\right) \sqrt{\frac{g}{4\left(h+\overline{c_{1}}\right)}} \partial_{X_{1}}^{3} r-\left(h+\overline{c_{1}}\right) \sqrt{\frac{g}{h+\overline{c_{1}}}} \partial_{X_{1}}^{-1}\left(\partial_{X_{2}}^{2} r\right), \\
\partial_{T} s= & \partial_{X_{1}} \delta_{s}\left(H-c_{0} I\right) / \varepsilon^{5}=0,
\end{aligned}
$$

which is accurate up to terms of order $\mathcal{O}\left(\varepsilon^{2}\right)$. Equation (4.41) corresponds to the KP equation for a rapidly varying bottom topography in three dimensions, while equation (4.42) implies that in the present approximation there is no change in $s$, at least over time intervals $T \in\left[-T_{0}(\varepsilon), T_{0}(\varepsilon)\right]$, with $T_{0}(\varepsilon)=\mathcal{O}\left(\varepsilon^{-2}\right)$.

\section{Bottom topography with multiple spatial scales}

\subsection{The two-dimensional Boussinesq regime}

We consider the Boussinesq scaling (4.1) but now allow the bottom to vary both on a length scale $\mathcal{O}(1)$ as well as on a slowly varying scale, namely $\beta=\beta(x, X)$ where $X=\varepsilon x$. Again, no assumption is made on the amplitude of $\beta$. It also may be possible that more than one slow scale behavior is present, whereupon we write $\beta=\beta(x, X ; \varepsilon)$. We will make explicit the behavior of multiple scales. As before, the effort in the asymptotic expansion is almost 
entirely in examining the Dirichlet-Neumann operator, giving the result that

$$
\begin{aligned}
D L(\beta)= & -\varepsilon D_{x} B_{0}(\beta) \beta D_{X} \\
& -\varepsilon^{2} D_{X} B_{0}(\beta) \beta D_{X}+\varepsilon^{2} D_{x} B_{0}(\beta) b \sinh \left(b D_{x}\right) D_{X} B_{0}(\beta) \beta D_{X} \\
& +\varepsilon^{3} \frac{1}{2} h^{2} D_{x} B_{0}(\beta) \beta D_{X}^{3}-\varepsilon^{3} \frac{1}{6} D_{x} B_{0}(\beta) \beta^{3} D_{X}^{3} \\
& +\varepsilon^{3} \frac{1}{2} D_{x} B_{0}(\beta) b^{2} \cosh \left(b D_{x}\right) D_{X}^{2} B_{0}(\beta) \beta D_{X} \\
& +\varepsilon^{3} D_{X} B_{0}(\beta) b \sinh \left(b D_{x}\right) D_{X} B_{0}(\beta) \beta D_{X} \\
& -\varepsilon^{3} D_{x} B_{0}(\beta) b \sinh \left(b D_{x}\right) D_{X} B_{0}(\beta) b \sinh \left(b D_{x}\right) D_{X} B_{0}(\beta) \beta D_{X} \\
& +\varepsilon^{4} \frac{1}{2} h^{2} D_{X} B_{0}(\beta) \beta D_{X}^{3}-\varepsilon^{4} \frac{1}{6} D_{X} B_{0}(\beta) \beta^{3} D_{X}^{3} \\
& +\varepsilon^{4} \frac{1}{2} D_{X} B_{0}(\beta) b^{2} \cosh \left(b D_{x}\right) D_{X}^{2} B_{0}(\beta) \beta D_{X} \\
& -\varepsilon^{4} \frac{1}{2} h^{2} D_{x} B_{0}(\beta) b \sinh \left(b D_{x}\right) D_{X} B_{0}(\beta) \beta D_{X}^{3} \\
& +\varepsilon^{4} \frac{1}{6} D_{x} B_{0}(\beta) b \sinh \left(b D_{x}\right) D_{X} B_{0}(\beta) \beta^{3} D_{X}^{3} \\
& +\varepsilon^{4} \frac{1}{6} D_{x} B_{0}(\beta) b^{3} \sinh \left(b D_{x}\right) D_{X}^{3} B_{0}(\beta) \beta D_{X} \\
& -\varepsilon^{4} \frac{1}{2} D_{x} B_{0}(\beta) b \sinh \left(b D_{x}\right) D_{X} B_{0}(\beta) b^{2} \cosh \left(b D_{x}\right) D_{X}^{2} B_{0}(\beta) \beta D_{X} \\
& -\varepsilon^{4} \frac{1}{2} D_{x} B_{0}(\beta) b^{2} \cosh \left(b D_{x}\right) D_{X}^{2} B_{0}(\beta) b \sinh \left(b D_{x}\right) D_{X} B_{0}(\beta) \beta D_{X} \\
& -\varepsilon^{4} D_{X} B_{0}(\beta) b \sinh \left(b D_{x}\right) D_{X} B_{0}(\beta) b \sinh \left(b D_{x}\right) D_{X} B_{0}(\beta) \beta D_{X} \\
& +\varepsilon^{4} D_{x} B_{0}(\beta) b \sinh \left(b D_{x}\right) D_{X} B_{0}(\beta) b \sinh \left(b D_{x}\right) \\
& \times D_{X} B_{0}(\beta) b \sinh \left(b D_{x}\right) D_{X} B_{0}(\beta) \beta D_{X}+\mathcal{O}\left(\varepsilon^{5}\right)
\end{aligned}
$$

in the resulting expansion of $G^{(0)}$ in (4.3), and in the expansion of the term $G^{(1)}$ in $(4.4)$, one finds that

$$
\begin{aligned}
D \tanh (h D) \eta D L(\beta)= & -\varepsilon^{3} D_{x}^{2} \tanh \left(h D_{x}\right) B_{0}(\beta) \beta \eta^{\prime}(X) D_{X} \\
& +\varepsilon^{4} D_{x}^{2} \tanh \left(h D_{x}\right) B_{0}(\beta) b \sinh \left(b D_{x}\right) \eta^{\prime}(X) D_{X} B_{0}(\beta) \beta D_{X} \\
& -\varepsilon^{4} D_{x} \tanh \left(h D_{x}\right) \eta^{\prime}(X) D_{X} B_{0}(\beta) \beta D_{X} \\
& -\varepsilon^{4} h D_{x}^{2} \operatorname{sech}\left(h D_{x}\right)^{2} D_{X} B_{0}(\beta) \beta \eta^{\prime}(X) D_{X} \\
& -\varepsilon^{4} D_{x} \tanh \left(h D_{x}\right) D_{X} B_{0}(\beta) \beta \eta^{\prime}(X) D_{X}+\mathcal{O}\left(\varepsilon^{5}\right),
\end{aligned}
$$

and one finds that

$$
\begin{aligned}
D L(\beta) \eta D L(\beta)= & \varepsilon^{3} D_{x} B_{0}(\beta) \sinh \left(\beta D_{x}\right) D_{x} \operatorname{sech}\left(h D_{x}\right) B_{0}(\beta) \beta \eta^{\prime}(X) D_{X} \\
- & \varepsilon^{4} D_{x} B_{0}(\beta) \sinh \left(\beta D_{x}\right) D_{x} \operatorname{sech}\left(h D_{x}\right) \\
& \times B_{0}(\beta) b \sinh \left(b D_{x}\right) \eta^{\prime}(X) D_{X} B_{0}(\beta) \beta D_{X} \\
+ & \varepsilon^{4} D_{x} B_{0}(\beta) \sinh \left(\beta D_{x}\right) \operatorname{sech}\left(h D_{x}\right) \eta^{\prime}(X) D_{X} B_{0}(\beta) \beta D_{X} \\
- & \varepsilon^{4} h D_{x} B_{0}(\beta) \sinh \left(\beta D_{x}\right) D_{x} \tanh \left(h D_{x}\right) \\
& \times \operatorname{sech}\left(h D_{x}\right) D_{X} \eta^{\prime}(X) B_{0}(\beta) \beta D_{X} \\
+ & \varepsilon^{4} D_{x} B_{0}(\beta) \beta \cosh \left(\beta D_{x}\right) D_{x} \operatorname{sech}\left(h D_{x}\right) \\
& \times D_{X} \eta^{\prime}(X) B_{0}(\beta) \beta D_{X} \\
- & \varepsilon^{4} D_{x} B_{0}(\beta) b \sinh \left(b D_{x}\right) D_{X} B_{0}(\beta) \sinh \left(\beta D_{x}\right) \\
& \times D_{x} \operatorname{sech}\left(h D_{x}\right) B_{0}(\beta) \beta \eta^{\prime}(X) D_{X} \\
+ & \varepsilon^{4} D_{X} B_{0}(\beta) \sinh \left(\beta D_{x}\right) D_{x} \operatorname{sech}\left(h D_{x}\right) \\
& \times B_{0}(\beta) \beta \eta^{\prime}(X) D_{X}+\mathcal{O}\left(\varepsilon^{5}\right) .
\end{aligned}
$$


For simplicity of notation, the quantities $\beta$ and $b$ are written without any independent variables in (5.1), (5.2) and (5.3) but recall that $\beta=\beta(x, X)$ and $b=b(x, X)=\beta(x, X)-h$ are functions of multiple spatial scales.

Retaining explicit calculations of terms of up to order $\mathcal{O}\left(\varepsilon^{3}\right)$, the Hamiltonian reads

$$
\begin{aligned}
\frac{1}{\varepsilon^{3}} H=\frac{1}{2} & \int-\varepsilon^{-1} D_{x} B_{0}(\beta) \beta \xi^{\prime}(X) D_{X} \xi^{\prime}(X)+g \eta^{\prime 2}(X) \\
& -\left(1-D_{x} B_{0}(\beta) b \sinh \left(b D_{x}\right)\right) \xi^{\prime}(X) D_{X} B_{0}(\beta) \beta D_{X} \xi^{\prime}(X) \\
& +h \xi^{\prime}(X) D_{X}^{2} \xi^{\prime}(X) d X+\mathcal{O}\left(\varepsilon^{1}\right) .
\end{aligned}
$$

Again, the lemma of scale separation allows us to write

$$
\frac{1}{\varepsilon^{3}} H=\frac{1}{2} \int g \eta^{\prime 2}(X)-\left(h+\overline{c_{1}}(X)\right)\left(D_{X} \xi^{\prime}\right)^{2} d X+\mathcal{O}(\varepsilon),
$$

where $c_{1}=-B_{0}(\beta) \beta$. Dropping the primes, the corresponding equations of motion are given in terms of $u=\partial_{X} \xi$ by

$$
\partial_{t} \eta=-\partial_{X}\left(\left(h+\overline{c_{1}}\right) u\right), \quad \partial_{t} u=-g \partial_{X} \eta .
$$

In this form, equations (5.6) only differ from (4.14) by the $X$-dependence of the coefficient $\overline{c_{1}}$. Higher order corrections can be derived in a similar way. Keeping terms up to order $\mathcal{O}\left(\varepsilon^{5}\right)$ in the Hamiltonian, we have

$$
\begin{aligned}
& \frac{1}{\varepsilon^{3}} H=\frac{1}{2} \int g \eta^{\prime 2}(X)-\left(h+\overline{c_{1}}(X)\right)\left(D_{X} \xi^{\prime}\right)^{2}-\varepsilon \overline{c_{2}}(X)\left(D_{X} \xi^{\prime}\right)^{2}-\varepsilon^{2} \overline{c_{3}}(X)\left(D_{X} \xi^{\prime}\right)^{2} \\
& \quad+\varepsilon^{2}\left(\overline{c_{4}}(X)+\frac{1}{3} h^{3}\right)\left(D_{X} \xi^{\prime}\right) D_{X}^{3} \xi^{\prime}-\varepsilon^{2} \overline{c_{5}}(X)\left(D_{X}^{2} \xi^{\prime}\right)^{2}-\varepsilon^{2}\left(1+\overline{c_{6}}(X)\right) \eta^{\prime}(X)\left(D_{X} \xi^{\prime}\right)^{2} d X \\
& \quad+\mathcal{O}\left(\varepsilon^{3}\right),
\end{aligned}
$$

with

$$
\begin{aligned}
c_{2}=B_{0}(\beta) & b \sinh \left(b D_{x}\right) D_{X}\left(B_{0}(\beta) \beta\right)-\frac{1}{2} D_{X}\left(B_{0}(\beta) b \sinh \left(b D_{x}\right) B_{0} \beta\right), \\
c_{3}= & \frac{1}{2} B_{0}(\beta) b^{2} \cosh \left(b D_{x}\right) D_{X}^{2}\left(B_{0}(\beta) \beta\right) \\
& +D_{X}\left(B_{0}(\beta) b \sinh \left(b D_{x}\right)\right) B_{0}(\beta) b \sinh \left(b D_{x}\right) D_{X}\left(B_{0}(\beta) \beta\right) \\
& -\frac{1}{2} D_{X}\left(B_{0}(\beta) b^{2} \cosh \left(b D_{x}\right) D_{X}\left(B_{0}(\beta) \beta\right)\right. \\
& +D_{X}\left(B_{0}(\beta) b \sinh \left(b D_{x}\right)\right) B_{0}(\beta) b \sinh \left(b D_{x}\right) B_{0}(\beta) \beta \\
& \left.+B_{0}(\beta) b \sinh \left(b D_{x}\right) B_{0}(\beta) b \sinh \left(b D_{x}\right) D_{X}\left(B_{0}(\beta) \beta\right)\right), \\
& c_{4}=-\frac{1}{2} h^{2} B_{0}(\beta) \beta+\frac{1}{6} B_{0}(\beta) \beta^{3}-\frac{1}{2} B_{0}(\beta) b^{2} \beta \\
& c_{5}=B_{0}(\beta) b \sinh \left(b D_{x}\right) B_{0}(\beta) b \sinh \left(b D_{x}\right) B_{0}(\beta) \beta,
\end{aligned}
$$

and

$$
c_{6}=-B_{0}(\beta) \sinh \left(\beta D_{x}\right) D_{x} \operatorname{sech}\left(h D_{x}\right) B_{0}(\beta) \beta .
$$

The equations of motion given by this approximation are

$$
\begin{aligned}
\partial_{t} \eta & =-\partial_{X}\left(\left(\bar{h}(X)+\varepsilon^{2}\left(1+\overline{c_{6}}\right) \eta\right) u\right)-\varepsilon^{2} \partial_{X}^{2}\left(\left(\frac{1}{3} h^{3}+\overline{c_{4}}+\overline{c_{5}}\right) \partial_{X} u\right) \\
\partial_{t} u & =-g \partial_{X} \eta-\frac{1}{2} \varepsilon^{2} \partial_{X}\left(\left(1+\overline{c_{6}}\right) u^{2}\right)
\end{aligned}
$$


where the effective depth is expressed by $\bar{h}(X)=h+\overline{c_{1}}+\varepsilon \overline{c_{2}}+\varepsilon^{2} \overline{c_{3}}+\frac{1}{2} \varepsilon^{2}\left(\partial_{X}^{2} \overline{c_{4}}\right)$.

Recall that the coefficients $\overline{c_{i}}$ are $X$-dependent. One can see that the presence of a slowly varying bottom topography introduces additional dispersive and nonlinear terms in the evolution equation of $\eta$. It can be seen from the form of the terms of order $\mathcal{O}\left(\varepsilon^{5}\right)$ in the expansion of the rescaled Dirichlet-Neumann operator (5.1) that there are nonzero terms in the approximate Hamiltonian (5.7) of order $\mathcal{O}\left(\varepsilon^{6}\right)$, and therefore the time interval of validity of these equations is less than in the case of a bottom with no variations on a slow spatial scale. The most pessimistic view states that the error terms implied in the approximate long wave equations (5.13) are of order $\mathcal{O}\left(\varepsilon^{3}\right)$, and therefore it affords the possibility that over time intervals longer than $T(\varepsilon)=o\left(\varepsilon^{-1}\right)$ the error can grow to compete with the terms retained in the system of equations (5.13).

\subsection{The three-dimensional Boussinesq regime}

In the three dimensional situation, in cases where the bottom varies both on a length scale $\mathcal{O}(1)$ as well as on a slowly varying scale in both horizontal directions (i.e. $\beta=$ $\left.\beta\left(x_{1}, X_{1}, x_{2}, X_{2}\right)\right)$, we find up to order $\mathcal{O}\left(\varepsilon^{3}\right)$ and $\mathcal{O}\left(\varepsilon^{5}\right)$, respectively

$$
H=\frac{\varepsilon^{3}}{2} \int g \eta^{\prime 2}-\left(h+\overline{c_{1}}\right)\left(D_{X_{1}} \xi^{\prime}\right)^{2} d X_{1} d X_{2}+\mathcal{O}\left(\varepsilon^{4}\right),
$$

where $c_{1}=-B_{0}(\beta) \beta$ and

$$
\begin{aligned}
H=\frac{\varepsilon^{3}}{2} \int g \eta^{\prime 2}-\left(h+\overline{c_{1}}\right)\left(D_{X_{1}} \xi^{\prime}\right)^{2}-\varepsilon \overline{c_{2}}\left(D_{X_{1}} \xi^{\prime}\right)^{2} \\
\quad-\varepsilon^{2} \overline{c_{3}}\left(D_{X_{1}} \xi^{\prime}\right)^{2}+\varepsilon^{2}\left(\overline{c_{4}}+\frac{1}{3} h^{3}\right)\left(D_{X_{1}} \xi^{\prime}\right) D_{X_{1}}^{3} \xi^{\prime}-\varepsilon^{2} \overline{c_{5}}\left(D_{X_{1}}^{2} \xi^{\prime}\right)^{2} \\
\quad-\varepsilon^{2}\left(1+\overline{c_{6}}\right) \eta^{\prime}\left(D_{X_{1}} \xi^{\prime}\right)^{2}-\varepsilon^{2}\left(h+\overline{c_{1}}\right)\left(D_{X_{2}} \xi^{\prime}\right)^{2} \\
\quad+\varepsilon^{2} \overline{c_{7}}\left(D_{X_{1}} \xi^{\prime}\right)\left(D_{X_{1}} D_{X_{2}} \xi^{\prime}\right) d X_{1} d X_{2}+\mathcal{O}\left(\varepsilon^{6}\right)
\end{aligned}
$$

with

$$
\begin{gathered}
c_{2}=B_{0}(\beta) b \sinh (b|D|) D_{x_{1}}|D|^{-1} D_{X_{1}}\left(B_{0}(\beta) \beta\right) \\
-\frac{1}{2} D_{X_{1}}\left(B_{0}(\beta) b \sinh (b|D|) D_{x_{1}}|D|^{-1} B_{0} \beta\right) \\
c_{3}=\quad D_{X_{1}}\left(B_{0}(\beta) b \sinh (b|D|)\right) D_{x_{1}}|D|^{-1} B_{0}(\beta) b \sinh (b|D|) D_{x_{1}}|D|^{-1} D_{X_{1}}\left(B_{0}(\beta) \beta\right) \\
+\frac{1}{2} B_{0}(\beta) b^{2} \cosh (b|D|) D_{x_{1}}^{2}|D|^{-2} D_{X_{1}}^{2}\left(B_{0}(\beta) \beta\right) \\
+\frac{1}{2} B_{0}(\beta) b \sinh (b|D|)\left(1-D_{x_{1}}^{2}|D|^{-2}\right)|D|^{-1} D_{X_{1}}^{2}\left(B_{0}(\beta) \beta\right) \\
+B_{0}(\beta) b \sinh (b|D|) D_{x_{2}}|D|^{-1} D_{X_{2}}\left(B_{0}(\beta) \beta\right) \\
+\frac{1}{2} D_{X_{1}}\left(B_{0}(\beta) b^{2} \cosh (b|D|) D_{x_{1}}^{2}|D|^{-2} D_{X_{1}}\left(B_{0}(\beta) \beta\right)\right. \\
+B_{0}(\beta) b \sinh (b|D|)\left(1-D_{x_{1}}^{2}|D|^{-2}\right)|D|^{-1} D_{X_{1}}\left(B_{0}(\beta) \beta\right) \\
+D_{X_{1}}\left(B_{0}(\beta) b \sinh (b|D|)\right) D_{x_{1}}|D|^{-1} B_{0}(\beta) b \sinh (b|D|) D_{x_{1}}|D|^{-1} B_{0}(\beta) \beta \\
\left.+B_{0}(\beta) b \sinh (b|D|) D_{x_{1}}|D|^{-1} B_{0}(\beta) b \sinh (b|D|) D_{x_{1}}|D|^{-1} D_{X_{1}}\left(B_{0}(\beta) \beta\right)\right) \\
\quad \\
c_{4}=\quad-\frac{1}{2} h^{2} B_{0}(\beta) \beta+\frac{1}{6} B_{0}(\beta) \beta^{3}-\frac{1}{2} B_{0}(\beta) b^{2} \cosh (b|D|) D_{x_{1}}^{2}|D|^{-2} B_{0}(\beta) \beta \\
\quad-\frac{1}{2} B_{0}(\beta) b \sinh (b|D|)\left(1-D_{x_{1}}^{2}|D|^{-2}\right)|D|^{-1} B_{0}(\beta) \beta
\end{gathered}
$$




$$
\begin{aligned}
& c_{5}=B_{0}(\beta) b \sinh (b|D|) D_{x_{1}}|D|^{-1} B_{0}(\beta) b \sinh (b|D|) D_{x_{1}}|D|^{-1} B_{0}(\beta) \beta, \\
& c_{6}=-B_{0}(\beta) \sinh (\beta|D|) D_{x_{1}}^{2}|D|^{-1} \operatorname{sech}(h|D|) B_{0}(\beta) \beta \\
& -B_{0}(\beta) \sinh (\beta|D|) D_{x_{1}} D_{x_{2}}|D|^{-1} \operatorname{sech}(h|D|) B_{0}(\beta) \beta,
\end{aligned}
$$

and

$$
c_{7}=-i B_{0}(\beta) b \sinh (b|D|) D_{x_{2}}|D|^{-1} B_{0}(\beta) \beta .
$$

The corresponding approximate equations of motion to lowest order are,

$$
\begin{aligned}
\partial_{t} \eta= & -\partial_{X_{1}}\left(\left(\bar{h}\left(X_{1}, X_{2}\right)+\varepsilon^{2}\left(1+\overline{c_{6}}\right) \eta\right) \partial_{X_{1}} \xi\right)-\varepsilon^{2} \partial_{X_{1}}^{2}\left(\left(\overline{c_{4}}+\frac{1}{3} h^{3}+\overline{c_{5}}\right) \partial_{X_{1}}^{2} \xi\right) \\
& -\varepsilon^{2} \partial_{X_{2}}\left(\left(h+\overline{c_{1}}\right) \partial_{X_{2}} \xi\right) \\
\partial_{t} \xi= & -g \eta-\frac{1}{2} \varepsilon^{2}\left(1+\overline{c_{6}}\right)\left(\partial_{X_{1}} \xi\right)^{2}
\end{aligned}
$$

where the effective depth in this situation is given by the expression

$$
\bar{h}=h+\overline{c_{1}}+\varepsilon \overline{c_{2}}+\varepsilon^{2} \overline{c_{3}}+\frac{1}{2} \varepsilon^{2}\left(\partial_{X_{1}}^{2} \overline{c_{4}}\right)-\frac{1}{2} \varepsilon^{2}\left(\partial_{X_{2}} \overline{c_{7}}\right) .
$$

Recall that all coefficients $\overline{c_{i}}$ depend on $X_{1}$ and $X_{2}$.

\subsection{Unidirectional equations in a regime with multiple spatial scales}

In order to derive a unidirectional equation in the Boussinesq regime with multiple spatial scales, we extend our analysis by considering the more general case where $\beta=\beta(x, X ; \varepsilon)$. The equations of motion consist of a system of coupled equations for the right- and leftmoving components. The coupling at first order, which determines the role of wave scattering, is measured by the slope of the effective depth in the problem. Two typical examples of multiscale dependence for the bottom are

$$
\begin{aligned}
& \beta=\beta(x, \tilde{X}), \quad \tilde{X}=\varepsilon^{\alpha} X=\varepsilon^{\alpha+1} x, \\
& \beta=\beta_{0}(x)+\varepsilon^{\gamma} \beta_{1}(x, \tilde{X}), \quad \tilde{X}=\varepsilon^{\delta} X=\varepsilon^{\delta+1} x,
\end{aligned}
$$

where $\alpha, \gamma, \delta \geq 0$. The parameters $\alpha$ in (5.23) and $\gamma+\delta$ in (5.24) provide a measure for the steepness of the slowly varying bottom. This is the regime in which the bottom perturbation varies over an even longer length scale than the wave motion represented by $\eta(X)$ and $\xi(X)$. To illustrate the problem, we will present the analysis only in the case (5.23) which was also considered by E. van Groesen \& S.R. Pudjaprasetya [GP].

At the second order of approximation, the Hamiltonian (5.7) is given by

$$
\begin{aligned}
H=\frac{\varepsilon^{3}}{2} & \int g \eta^{\prime}(X)^{2}+\left(h+\overline{c_{1}}(\tilde{X})+\varepsilon^{\alpha+1} \overline{c_{2}}(\tilde{X})+\varepsilon^{2 \alpha+2} \overline{c_{3}}(\tilde{X})\right. \\
& \left.+\frac{1}{2} \varepsilon^{2 \alpha+2}\left(\partial_{\tilde{X}}^{2} \overline{c_{4}}(\tilde{X})\right)\right) u^{\prime}(X)^{2} \\
& -\varepsilon^{2}\left(\frac{1}{3} h^{3}+\overline{c_{4}}(\tilde{X})+\overline{c_{5}}(\tilde{X})\right)\left(\partial_{X} u^{\prime}(X)\right)^{2} \\
& +\varepsilon^{2}\left(1+\overline{c_{6}}(\tilde{X})\right) \eta^{\prime}(X) u^{\prime}(X)^{2} d X+\mathcal{O}\left(\varepsilon^{5+\alpha}\right) .
\end{aligned}
$$

Making the change of variables

$$
\eta^{\prime}=\sqrt[4]{\frac{\bar{h}(\tilde{X})}{4 g}}(r+s), \quad u^{\prime}=\sqrt[4]{\frac{g}{4 \bar{h}(\tilde{X})}}(r-s)
$$


where the effective depth $\bar{h}$ is

$$
\bar{h}(\tilde{X})=h+\overline{c_{1}}(\tilde{X})+\varepsilon^{\alpha+1} \overline{c_{2}}(\tilde{X})+\varepsilon^{2 \alpha+2} \overline{c_{3}}(\tilde{X})+\frac{1}{2} \varepsilon^{2 \alpha+2} \partial_{\tilde{X}}^{2} \overline{c_{4}}(\tilde{X}),
$$

the Hamiltonian becomes

$$
\begin{aligned}
H=\frac{\varepsilon^{3}}{2} & \int \sqrt{g \bar{h}}\left(r^{2}+s^{2}\right)-\varepsilon^{2}\left(\frac{1}{3} h^{3}+\overline{c_{4}}+\overline{c_{5}}\right)\left[\left(\sqrt[4]{\frac{g}{4 \bar{h}}} \partial_{X} r\right)^{2}\right. \\
& \left.-2\left(\sqrt[4]{\frac{g}{4 \bar{h}}} \partial_{X} r\right)\left(\sqrt[4]{\frac{g}{4 \bar{h}}} \partial_{X} s\right)+\left(\sqrt[4]{\frac{g}{4 \bar{h}}} \partial_{X} s\right)^{2}\right] \\
& +\varepsilon^{2}\left(\frac{1+\overline{c_{6}}}{2}\right) \sqrt[4]{\frac{g}{4 \bar{h}}}\left(r^{3}-r^{2} s-r s^{2}+s^{3}\right) d X+o\left(\varepsilon^{5}\right) .
\end{aligned}
$$

The evolution equations for $r$ and $s$ are now written as

$$
\partial_{t}\left(\begin{array}{c}
r \\
s
\end{array}\right)=\frac{1}{\varepsilon^{3}}\left(\begin{array}{cc}
-\partial_{X} & \frac{1}{4} \varepsilon^{\alpha}\left(\partial_{\tilde{X}} \log (\bar{h})\right) \\
-\frac{1}{4} \varepsilon^{\alpha}\left(\partial_{\tilde{X}} \log (\bar{h})\right) & \partial_{X}
\end{array}\right)\left(\begin{array}{c}
\delta_{r} H \\
\delta_{s} H
\end{array}\right) .
$$

The solutions $r$ and $s$ correspond to predominantly right- and left-moving wave motions, respectively. The presence of the non-diagonal terms $\frac{1}{4} \varepsilon^{\alpha} \partial_{\tilde{X}} \log (\bar{h}(\tilde{X}))$ in $(5.29)$ is a consequence of the bottom variations and gives rise to the effect of wave scattering. Note that, unlike the case of a rapidly varying bottom topography, we do not subtract the momentum integral from the Hamiltonian in order to transform to a moving reference frame, since momentum is not a conserved quantity when the depth is variable.

In case that $\alpha=0$, the result (5.29) is the following system of two coupled KdV-like equations;

$$
\begin{aligned}
\partial_{t} r= & -\partial_{X}\left(C_{0}(X) r+\varepsilon^{2} \partial_{X}\left(C_{1}(X)\left(\partial_{X} r-\partial_{X} s\right)\right)+\varepsilon^{2} C_{2}(X)\left(3 r^{2}-2 r s-s^{2}\right)\right) \\
& +S(X)\left(C_{0}(X) s-\varepsilon^{2} \partial_{X}\left(C_{1}(X)\left(\partial_{X} r-\partial_{X} s\right)\right)+\varepsilon^{2} C_{2}(X)\left(-r^{2}-2 r s+3 s^{2}\right)\right), \\
\partial_{t} s= & \partial_{X}\left(C_{0}(X) s-\varepsilon^{2} \partial_{X}\left(C_{1}(X)\left(\partial_{X} r-\partial_{X} s\right)\right)+\varepsilon^{2} C_{2}(X)\left(-r^{2}-2 r s+3 s^{2}\right)\right) \\
& -S(X)\left(C_{0}(X) r+\varepsilon^{2} \partial_{X}\left(C_{1}(X)\left(\partial_{X} r-\partial_{X} s\right)\right)+\varepsilon^{2} C_{2}(X)\left(3 r^{2}-2 r s-s^{2}\right)\right) .
\end{aligned}
$$

The coefficients are defined as

$$
\begin{aligned}
& C_{0}(X)=\sqrt{g \bar{h}(\tilde{X})}, \quad C_{1}(X)=\left(\frac{1}{3} h^{3}+\overline{c_{4}}+\overline{c_{5}}\right) \sqrt{\frac{g}{4 \bar{h}}}, \\
& C_{2}(X)=\frac{1}{4}\left(1+\overline{c_{6}}\right) \sqrt[4]{\frac{g}{4 \bar{h}}}, \quad S(X)=\frac{1}{4}\left(\partial_{\tilde{X}} \log (\bar{h})\right) .
\end{aligned}
$$

In (5.30) and (5.31), the expression for the effective linear phase speed is $C_{0}(X)$; we note that this includes higher order terms in $\varepsilon$. Because of the strong coupling between the wave fields $r(X, t)$ and $s(X, t)$, these two do not separate into independent solutions for a unidirectional regime.

When $\alpha>0$, and when the initial data $r_{0}(X)$ and $s_{0}(X)$ are functions which are localized spatially, the analysis divides into several cases. 
Case (1): When $\alpha \geq 2$, corresponding to very mild slopes, and initial conditions $s_{0}=\mathcal{O}\left(\varepsilon^{2}\right)$, one rescales $s=\varepsilon^{2} s^{\prime}$ and $r=r^{\prime}$ in equations (5.30), (5.31). Dropping terms of higher order, we have

$$
\begin{aligned}
& \partial_{t} r^{\prime}=-\partial_{X}\left(C_{0}(X) r^{\prime}+\varepsilon^{2}\left(\partial_{X}\left(C_{1}(X) \partial_{X} r^{\prime}\right)+3 C_{2}(X) r^{2}\right)\right) \\
& \partial_{t} s^{\prime}=\partial_{X}\left(C_{0}(X) s^{\prime}-\partial_{X}\left(C_{1}(X) \partial_{X} r^{\prime}\right)-C_{2}(X) r^{\prime 2}\right)-\varepsilon^{\alpha-2} S(X) C_{0}(X) r^{\prime} .
\end{aligned}
$$

This system consists of an equation in closed form for $r^{\prime}(X, t)$, which is a $\mathrm{KdV}$ equation with slowly varying coefficients representing a principally right-moving wave field, and a second equation for a reflected wave $s^{\prime}(X, t)$. The solution $s^{\prime}(X, t)$ is recovered from $r^{\prime}(X, t)$ by quadrature along left-moving characteristics, defined by $\dot{X}=-C_{0}(X)$.

This system of equations describing $r^{\prime}(X, t)$ is a valid asymptotic regime at least over time intervals of order $T(\varepsilon)=o\left(\varepsilon^{-1}\right)$, the same time interval as for the Boussinesq system (5.13), but a strictly shorter time interval than the case of the KdV equation with constant coefficients (as arising with a flat or a rapidly varying bottom without slow spatial variations). This is due in part to the nature of the error from truncation of the Hamiltonian. Furthermore, over such time intervals the scattering component $s^{\prime}(X, t)$ remains bounded. This latter fact is true because initial conditions $r_{0}^{\prime}(X)$ which is spatially localized (that is, essentially supported in a neighborhood of diameter $\mathcal{O}(1))$ gives rise to solutions $r^{\prime}(X, t)$ which move essentially to the right. The quadrature for the scattering component $s^{\prime}(X, t)$ is along left-moving characteristics, which encounter regions in which $r^{\prime}(X, t)$ is of significant amplitude only over intervals of length $\mathcal{O}(1)$. Therefore $s^{\prime}(X, t)$ does not grow by an amount larger than $\mathcal{O}(1)$, even for quadrature over long time intervals, as much as $T(\varepsilon)=o\left(\varepsilon^{-1}\right)$. This will not be the case over longer intervals, for initial conditions which is not spatially localized, for instance in case the inital conditions are periodic.

Case (2): When $3 / 2 \leq \alpha<2$ and the initial conditions satisfy $s_{0}=\mathcal{O}\left(\varepsilon^{\alpha}\right)$, we obtain equations for $r(X, t)=r^{\prime}(X, t)$ and $s(X, t)=\varepsilon^{\alpha} s^{\prime}(X, t)$ which are again in the form of a $\mathrm{KdV}$ equation coupled to an equation for the left-moving scattered field;

$$
\begin{aligned}
& \partial_{t} r^{\prime}=-\partial_{X}\left(C_{0}(X) r^{\prime}+\varepsilon^{2}\left(\partial_{X}\left(C_{1}(X) \partial_{X} r^{\prime}\right)+3 C_{2}(X) r^{2}\right)\right) \\
& \partial_{t} s^{\prime}=\partial_{X}\left(C_{0}(X) s^{\prime}-\varepsilon^{2-\alpha} \partial_{X}\left(C_{1}(X) \partial_{X} r^{\prime}\right)-\varepsilon^{2-\alpha} C_{2}(X) r^{\prime 2}\right)-S(X) C_{0}(X) r^{\prime}
\end{aligned}
$$

Equation (5.33) is a $\mathrm{KdV}$ equation in $r$ with variable coefficients. The evolution of the reflected component of the solution $s^{\prime}(X, t)$ is governed by (5.34). From the scaling, it is proportional to the slope of the bottom. As in the previous case, this scaling regime is valid for time intervals of order $T(\varepsilon)=o\left(\varepsilon^{-1}\right)$.

Case (3): When $1<\alpha<3 / 2$ and the initial conditions satisfy $s_{0}=\mathcal{O}\left(\varepsilon^{\alpha}\right)$, the system of equations describing $r^{\prime}(X, t)$ is still given by (5.33), but the error in truncation is potentially larger, and the time interval in which one is assured of the validity of the approximation is shorter, namely $T(\varepsilon)=o\left(\varepsilon^{2(1-\alpha)}\right)$.

Case (4): If $\alpha=1$ the basic equation for the right-moving component $r^{\prime}(X, t)$ is modified by a coupling term to the reflected component. The appropriate scaling is $r(X, t)=r^{\prime}(X, t)$ and $s(X, t)=\varepsilon s^{\prime}(X, t)$, giving rise to the system of equations

$$
\begin{aligned}
\partial_{t} r^{\prime}= & -\partial_{X}\left(C_{0}(X) r^{\prime}+\varepsilon^{2}\left(\partial_{X}\left(C_{1}(X) \partial_{X} r^{\prime}\right)+3 C_{2}(X) r^{\prime 2}\right)\right) \\
& +\varepsilon^{2} S(X) C_{0}(X) s^{\prime} \\
\partial_{t} s^{\prime}= & \partial_{X}\left(C_{0}(X) s^{\prime}\right)-S(X) C_{0}(X) r^{\prime} .
\end{aligned}
$$


The scaling regime gives a valid approximation for both $r^{\prime}(X, t)$ and $s^{\prime}(X, t)$, for time intervals of order $T(\varepsilon)=o\left(\varepsilon^{-1}\right)$ as in Case (1); it is of this length because of the increased accuracy given by inclusion of the extra coupling term, although we sacrifice the simplicity of a decoupled equation for $r^{\prime}(X, t)$. We also note that because of the coarser scaling in $s^{\prime}$ the permissible error is greater.

Case (5): Finally, if $0<\alpha \leq 1$, the evolutions of $r^{\prime}=r$ and $\varepsilon^{\alpha} s^{\prime}=s$ decouple only weakly, and over time intervals of order $\mathcal{O}\left(\varepsilon^{-2 \alpha}\right)$. A coarse description of the evolution is given by

$$
\begin{aligned}
\partial_{t} r^{\prime} & =-\partial_{X}\left(C_{0}(X) r^{\prime}\right) \\
\partial_{t} s^{\prime} & =\partial_{X}\left(C_{0}(X) s^{\prime}\right)-S(X) C_{0}(X) r^{\prime},
\end{aligned}
$$

where the error of truncation is $\mathcal{O}\left(\varepsilon^{2 \alpha}\right)$. Dispersion in the right-moving component is not as significant as scattering effects in this linear system, and therefore dispersive terms have been eliminated. Returning to the case $\alpha=0$ corresponds to the fully coupled system (5.30), (5.31).

Similar results can be obtained for a bottom topography of the form (5.24). In particular, the case $\gamma=2, \delta=0$ was examined by G. Whitham [W]. It should be noted that KdV-type equations for a slowly varying bottom in this setting have also been derived by several other authors, including R. S. Johnson [J], J. Miles [M] and A. Newell [N].

The present analysis can be extended to three dimensions in a straightforward manner. For brevity, we will only give one example. Assuming that the wave motion is primarily one-dimensional in the $x_{1}$-direction and that the bottom has the following dependence

$$
\beta=\beta\left(x_{1}, \tilde{X}_{1}, x_{2}, \tilde{X}_{2}\right),
$$

where $\tilde{X}_{1}=\varepsilon^{\alpha} X_{1}=\varepsilon^{\alpha+1} x_{1}$ and $\tilde{X}_{2}=\varepsilon^{2 \alpha} X_{2}=\varepsilon^{2 \alpha+2} x_{2}$, a three-dimensional analog of (5.32), for $\alpha \geq 2$ can be derived as

$$
\begin{aligned}
\partial_{t} r= & -\sqrt{g \bar{h}} \partial_{X_{1}} r-\varepsilon^{2} \sqrt{\frac{g}{4 \bar{h}}}\left(\overline{c_{4}}+\frac{1}{3} h^{3}+\overline{c_{5}}\right) \partial_{X_{1}}^{3} r \\
& -3 \varepsilon^{2} \sqrt[4]{\frac{g}{4 \bar{h}}}\left(\frac{1+\overline{c_{6}}}{2}\right) r \partial_{X_{1}} r-\varepsilon^{2} \sqrt{\frac{g}{4 \bar{h}}}\left(h+\overline{c_{1}}\right) \partial_{X_{1}}^{-1}\left(\partial_{X_{2}}^{2} r\right),
\end{aligned}
$$

with $\bar{h}$ redefined as

$$
\bar{h}\left(\tilde{X}_{1}, \tilde{X}_{2}\right)=h+\overline{c_{1}}+\varepsilon^{\alpha+1} \overline{c_{2}}+\varepsilon^{2 \alpha+2} \overline{c_{3}}+\frac{1}{2} \varepsilon^{2 \alpha+2} \partial_{\tilde{X}_{1}}^{2} \overline{c_{4}}-\frac{1}{2} \varepsilon^{2 \alpha+2} \partial_{\tilde{X}_{2}} \overline{c_{7}} .
$$

Equation (5.38) can be viewed as the KP equation with slowly varying coefficients for very mild slopes of the bottom.

\section{Conclusions}

We study the long-wave asymptotic regime for water waves in a fluid domain of variable depth as a perturbation problem for a Hamiltonian system depending on a small parameter. The formulation of the problem in terms of Zakharov's Hamiltonian, using an expression for the Dirichlet integral in terms of the Dirichlet-Neumann operator, is convenient for the analysis. When the bottom varies periodically on a short length scale, the motion of long wavelength solutions is essentially governed by a system related to the Boussinesq 
equation, whose effective coefficients are determined by homogenisation averages. In a regime emphasizing one-way propagation, the same conclusion holds for solutions of a KdV equation. Similar results hold in the three dimensional case, where bi-directional long wavelength motions are governed by a Boussinesq-like system, and one-way motions by a system closely related to the KP equations. Expressions for the coefficients are quite explicit, and typical cases are computed numerically. We find that (1) the linear wave speed of the long waves is slower for non-constant bottom variations, than that of a flat bottom with the same average depth. This recovers the result of $[R P]$ in two dimensions for a periodic bottom. (2) For bottom variations with fixed average depth, but which approach the shoaling limit, the effective coefficient of the nonlinear term dominates the effective coefficients of dispersive terms in the KdV equation. However this is significant only for very large variations, and over a large range of bottom perturbations the nonlinearity and the dispersive effects are quite well balanced. This serves to justify the use of the Boussinesq and $\mathrm{KdV}$ equations for a wide range of bottom topography. (3) The time scale of the effects of both nonlinearity and dispersion are significantly slower for large amplitude variations of the bottom (always considering the average depth fixed).

In cases in which the periodic variations of the bottom themselves vary on the long length scale, we derive a number of Hamiltonian PDEs with variable coefficients to describe the evolution of surface waves. Because of the presence of the variations in the bottom, there are additional terms in the long wave equations. Even at the lowest order in perturbation theory, terms are present which describe the linear effect of reflection of waves by the bottom topography. Denoting the effective depth by $\bar{h}(X)$, we show that the linear reflection from left-propagating to right-propagating modes (and vice versa) is proportional to $S=$ $\partial_{X} \log (\bar{h})$. This coefficient depends upon $X=\varepsilon x$, as do the resulting effective coefficients of dispersion and nonlinearity; it appears both in the two dimensional Boussinesq system and in its three dimensional analogue.

In the general case, the quantity $S(X)$ is of order $\mathcal{O}(1)$, and it does not make sense to seek special solutions whose motion is principally unidirectional; any such solution generates a substantial reflection in an $\mathcal{O}(1)$ length of time. However in cases in which $S=S(X ; \varepsilon)$ also depends upon the scaling parameter $\varepsilon$, such as when the long wavelength variations are small amplitude, or have small slope, or both, then $\partial_{X} \bar{h}(X ; \varepsilon) \sim \mathcal{O}\left(\varepsilon^{\alpha}\right)$, and it is possible to proceed further. Depending on the value of $\alpha$, we find five different regimes that can be described in detail.

Finally we would like to point out that the formulation of the Dirichlet-Neumann operator as given in this paper is suitable for the numerical simulation of the full equations of the water wave problem with bottom topography. The recursive expressions in terms of Fourier multipliers and of surface/bottom variations can be numerically evaluated using a spectral method with Fast Fourier Transforms. Numerical results will be presented in a separate paper.

\section{A Taylor expansions of $G(\beta, \eta)$ and $L(\beta)$}

In Section 2.2, we give a Taylor expansion of the Dirichlet-Neumann operator $G(\beta, \eta)$ in powers of $\eta$. The recursion formula for $G(\beta, \eta)$ is found to be the same as that for the case of a flat bottom, with the operator $L(\beta)$ being absorbed in the lowest order term $G^{(0)}$ of the recursion formula. Here we present an alternate formulation for $G(\beta, \eta)$ as a double series in both $\beta$ and $\eta$. This requires in particular a Taylor expansion of $L(\beta)$ in powers of $\beta$. 
Consider the Dirichlet-Neumann operator in the form $G(\beta, \eta)=\sum_{j, l} G_{j, l}(\beta, \eta)$ where the $G_{j, l}$ are homogeneous of degree $j$ and $l$ in powers of $\beta$ and $\eta$ respectively. The terms $G_{0, l}$ identify with the terms in the expansion of the Dirichlet-Neumann operator for a flat bottom. It is convenient to compute the coefficients $G_{j, 0}$ which correspond to a domain with a flat interface and a variable bottom topography. For this purpose let us consider the problem 2.6 with $\eta=0$. Its solution can be expressed in the form (2.9). By the definition of $G$, we have

$$
G(\beta, 0) \xi \equiv \partial_{y} \varphi(x, 0)=D \tanh (h D) \xi+D L(\beta) \xi
$$

Equivalently,

$$
G_{0,0}=D \tanh (h D), \quad G_{j, 0}=D \tanh (h D)+D L_{j}(\beta),
$$

where the $L_{j}(\beta)$ are the terms in the expansion of $L(\beta)$ in powers of $\beta$. To compute them explicitly, we write the Neumann condition at $y=-h+\beta(x)$

$$
\left(\partial_{y} \varphi-\partial_{x} \beta \partial_{x} \varphi\right)(x,-h+\beta)=0
$$

and expand the various terms in powers of $\beta$.

From the expression (2.9) for the function $\varphi(x, y)$ one calculates its derivatives. We now formally perform the Taylor expansions of the operators

$$
\begin{aligned}
& \left.D \sinh ((h+y) D)\right|_{y=-h+\beta}=\sum_{l \text { odd }} \frac{\beta^{l}}{l !} D^{l+1},\left.\quad D \cosh ((h+y) D)\right|_{y=-h+\beta}=\sum_{l \text { even }} \frac{\beta^{l}}{l !} D^{l+1}, \\
& \left.D \sinh (y D)\right|_{y=-h+\beta}=\sum_{l \text { even }} \frac{\beta^{l}}{l !}(-D)^{l+1} \sinh (h D)+\sum_{l \text { odd }} \frac{\beta^{l}}{l !}(-D)^{l+1} \cosh (h D), \\
& \left.D \cosh (y D)\right|_{y=-h+\beta}=\sum_{l \text { even }} \frac{\beta^{l}}{l !} D^{l+1} \cosh (h D)-\sum_{l \text { odd }} \frac{\beta^{l}}{l !} D^{l+1} \sinh (h D) .
\end{aligned}
$$

It is important to notice that in the above expansions, the operator $D$ does not act on $\beta$. Equation (A.3) is now rewritten as

$$
\begin{aligned}
& \sum_{l \text { odd }} \frac{\beta^{l}}{l !} \operatorname{sech}(h D) D^{l+1}+\sum_{l \text { even }} \frac{\beta^{l}}{l !} \cosh (h D) L(\beta)-\sum_{l \text { odd }} \frac{\beta^{l}}{l !} \sinh (h D) L(\beta) \\
& -i\left(\partial_{x} \beta\right)\left(\frac{\beta^{l}}{l !} \operatorname{sech}(h D) D^{l+1}+\sum_{l \text { odd }} \frac{\beta^{l}}{l !} \cosh (h D) L(\beta)-\sum_{l \text { even }} \frac{\beta^{l}}{l !} D^{l+1} \sinh (h D) L(\beta)\right) \\
& =0 .
\end{aligned}
$$

Identification of the terms in powers of $\beta$ leads at order $\mathcal{O}(1)$ to $L_{0}=0, L_{1}=-\operatorname{sech}(h D) \beta \operatorname{sech}(h D)$. $L_{2}=\operatorname{sech}(h D) \beta D \sinh (h D) L_{1}$. More generally, the recursion formula takes the form: for $j$ odd,

$L_{j}=-\operatorname{sech}(h D)\left[\frac{\beta^{j}}{j !} \operatorname{sech}(h D) D^{j}+\sum_{l=2, \text { even }}^{j-1} \frac{\beta^{l}}{l !} D^{l} \cosh (h D) L_{j-l}-\sum_{l=1, \text { odd }}^{j-2} \frac{\beta^{l}}{l !} D^{l} \sinh (h D) L_{j-l}\right]$,

and, for $j>0$ even,

$$
L_{j}=-\operatorname{sech}(h D)\left[\sum_{l=2, \text { even }}^{j-2} \frac{\beta^{l}}{l !} D^{l} \cosh (h D) L_{j-l}-\sum_{l=1, \text { odd }}^{j-1} \frac{\beta^{l}}{l !} D^{l} \sinh (h D) L_{j-l}\right] .
$$


We point out that the recursion formula given in (A.6) and (A.7) can be directly obtained by a Taylor expansion of the implicit formula for $L(\beta)$ in powers of $\beta$.

The recursion formula of $L(\beta)$ can be easily extended to three dimensions, taking the form: for $j$ odd,

$$
\begin{aligned}
L_{j}= & -\frac{D}{|D|} \operatorname{sech}(h|D|) \cdot\left[\frac{\beta^{j}}{j !} \operatorname{sech}(h|D|)|D|^{j-1} D+\sum_{l=2, \text { even }}^{j-1} \frac{\beta^{l}}{l !} \cosh (h|D|)|D|^{l-1} D L_{j-l}\right. \\
& \left.-\sum_{l=1, \text { odd }}^{j-2} \frac{\beta^{l}}{l !} \sinh (h|D|)|D|^{l-1} D L_{j-l}\right]
\end{aligned}
$$

and, for $j>0$ even,

$L_{j}=-\frac{D}{|D|} \operatorname{sech}(h|D|) \cdot\left[\sum_{l=2, \text { even }}^{j-2} \frac{\beta^{l}}{l !} \cosh (h|D|)|D|^{l-1} D L_{j-l}-\sum_{l=1, \text { odd }}^{j-1} \frac{\beta^{l}}{l !} \sinh (h|D|)|D|^{l-1} D L_{j-l}\right]$.

Putting together the expansions of $L(\beta)$ in powers of $\beta$ above and the expansions of $G(\beta, \eta)$ in terms of $L(\beta)$ and powers of $\eta$ as given in Section 2.3, one obtains an expression for the series expansion of the Dirichlet-Neumann operator as a double series in $\beta(x)$ and

$\eta(x)$. Using the fact that $G(\beta, \eta)$ is self-adjoint, one can write: for any $j>0$ and $l>0$ even,

$$
\begin{aligned}
G_{j, l} & =L_{j}|D|^{l-1} D \cdot \frac{\eta^{l}}{l !} D-\sum_{p=0, \text { even }}^{l-2}|D|^{l-p} \frac{\eta^{l-p}}{(l-p) !} G_{j, p} \\
& -\sum_{p=1, \text { odd }}^{l-1} G_{0,0}|D|^{l-p-1} \frac{\eta^{l-p}}{(l-p) !} G_{j, p}-\sum_{p=1, \text { odd }}^{l-1} \sum_{q=0}^{j-1} L_{j-q}|D|^{l-p} \frac{\eta^{l-p}}{(l-p) !} G_{q, p},\left(\mathrm{~A}^{-1}\right.
\end{aligned}
$$

and, for any $j>0$ and $l$ odd,

$$
\begin{aligned}
G_{j, l} & =-\sum_{p=1, \text { odd }}^{l-2}|D|^{l-p} \frac{\eta^{l-p}}{(l-p) !} G_{j, p}-\sum_{p=0, \text { even }}^{l-1} G_{0,0}|D|^{l-p-1} \frac{\eta^{l-p}}{(l-p) !} G_{j, p} \\
& -\sum_{p=0, \text { even }}^{l-1} \sum_{q=0}^{j-1} L_{j-q}|D|^{l-p} \frac{\eta^{l-p}}{(l-p) !} G_{q, p} .
\end{aligned}
$$

\section{References}

[BLP] A. Bensoussan, J.-L, Lions and G. Papanicolaou. 1978 Asymptotic analysis of periodic structures. Studies in Math. and its Applications, 5. North Holland.

[C] W. Craig. 1985 An existence theory for water waves and the Boussinesq and Korteweg-de Vries scaling limits. Comm. P. Diff. Eq. 8, pp. 787-1003.

[CG] W. Craig and M. Groves. 1994 Hamiltonian long-wave scaling limits of the water-wave problem. Wave Motion 19, pp. 367-389.

[CGK] W. Craig, P. Guyenne and H. Kalisch. Hamiltonian long wave expansions for free surfaces and interfaces. Manuscript in preparation. 
[CN] W. Craig and D. Nicholls. 2002 Traveling gravity water waves in two and three dimensions. European J. Mech. B - Fluids 21 no. 6, pp. 615-641.

[CS] W. Craig and C. Sulem. 1993 Numerical simulation of gravity waves. J. Comput. Phys. 108, pp. 73-83.

[CSS2] W. Craig, U. Schanz and C. Sulem. 1997 The modulation regime of threedimensional water waves and the Davey-Stewartson system. Ann. Inst. Henri Poincaré 14, pp. 615-667.

[CSS1] W. Craig, C. Sulem and P.-L. Sulem. 1992 Nonlinear modulation of gravity waves: a rigorous approach. Nonlinearity 5, pp. 497-552.

[GP] E. van Groesen and S.R. Pudjaprasetya. 1993 Uni-directional waves over slowly varying bottom. Part I: Derivation of KdV type equation. Wave Motion 18, pp. 345-370.

[J] R.S. Johnson. 1973 On the development of a solitary wave moving over an uneven bottom. Proc. Cambridge Philos. Soc. 73, pp. 183-203.

[K] J.T. Kirby. 1997 Nonlinear dispersive long waves in water of variable depth. in Gravity waves in water of finite depth (ed. J.N. Hunt). Advances in Fluid Mechanics, vol. 10, pp. 55-125.

[LY] Y. Liu and D.K.P. Yue. 1998 On generalized Bragg scattering of surface waves by bottom ripples. J. Fluid Mech. 356, pp. 297-356.

[ML] C.C. Mei and P.L.-F. Liu. 1993 Surface waves and coastal dynamics. Ann. Rev. Fluid Mech. 25, pp. 215-240.

[M] J.W. Miles. 1979 On the Korteweg-de Vries equation for a gradually varying channel. J. Fluid Mech. 91, pp. 181-190.

[NP] A. Nachbin and G. Papanicolaou. 1992 Water waves in channels of rapidly varying depth. J. Fluid Mech. 241, pp. 311-332.

[N] A.C. Newell. 1985 Solitons in Mathematics and Physics. SIAM, Philadelphia.

[RP] R. Rosales and G. Papanicolaou. 1983 Gravity waves in a channel with a rough bottom. Stud. Appl. Math. 68, pp. 89-102.

[S] R. Smith. 1998 An operator expansion formulation for nonlinear surface water waves over variable depth. J. Fluid Mech. 363, pp. 333-347.

[W] G.B. Whitham. 1974 Linear and nonlinear waves. Wiley-Interscience, New York.

[YL] S.B. Yoon and P.L.-F. Liu. 1994 A note on Hamiltonian for long water waves in varying depth. Wave Motion 20, pp. 359-370.

[Z] V. E. Zakharov. 1968 Stability of periodic waves of finite amplitude on the surface of a deep fluid. J. Appl. Mech. Tech. Phys. 9, pp. 1990-1994. 